\title{
Physiological Response and Productivity of Alfalfa to Potassium Foliar and Soil Applications under Saline Calcareous Soil Conditions
}

\author{
Mofeeda A. Seiam ${ }^{1}$, Mary E. Nashed ${ }^{2}$, M.E. El-Fayomy ${ }^{3}$ \\ ${ }^{1}$ Forage Crops Res. Department, field crop institute, A.R.C, Egypt Email, Mofeda seiam @ Gmail. Com. \\ ${ }^{2}$ Crop physiology Res. Department, Field crops institute, A.R.C., Egypt Email, mary. nashed..henen.4@ \\ Gmail. Com. \\ ${ }^{3}$ Soil, water and Environment Res. Institute. Land improvement and soil conservation department ARC, \\ Egypt. Email, D.ElFayoumy-7373@yahoo.com.
}

\begin{abstract}
A field experiment was conducted at Nubaria Agricultural Research Station, El-Behira Government, Egypt along the duration of 2015 to 2017 to study the physiological response and productivity of alfalfa (cv., Nubaria1) to seven potassium fertilization treatment of $0,28.6,57.14 \mathrm{Kg} / \mathrm{ha}$ as soil dressing, spraying with $1 \% \mathrm{~K}_{2} \mathrm{O}$ and $2 \% \mathrm{~K}_{2} \mathrm{O}$ alone or in combination with adding $28.6 \mathrm{Kg} \mathrm{K} 2 \mathrm{O} /$ ha under saline calcareous soil conditions. Experimental design was Randomized Complete Block design with four replications. Eighteen successive cuts were taken (Nine cuts /year), to estimate growth and physiological parameters, and alfalfa productivity.

Potassium fertilization treatments had significant effects on all studied traits in individual cuts except the $1^{\text {st }}$ and $9^{\text {th }}$ cuts in the first year and $2^{\text {nd }}$ cut in the second year for plant height, the $2^{\text {nd }}$ and $7^{\text {th }}$ cuts in the two years for dry matter\%, the $2^{\text {nd }}$ cut in the second year for dry forage $\mathrm{Kg} / \mathrm{m}^{2}$ and $1^{\text {st }}, 4^{\text {th }}$ and $5^{\text {th }}$ cuts for protein content in the first year.

In general, plant height, leaf steam ratio, green forage yield $\mathrm{Kg} / \mathrm{m}^{2}$, dry matter\%, dry forage yield $\mathrm{Kg} / \mathrm{m}^{2}$, relative water content, $\mathrm{K}^{+}$content, $\mathrm{K}^{+} / \mathrm{Na}^{+}$ratio and protein content increased significantly when plants received $28.6 \mathrm{~K} 2 \mathrm{O} / \mathrm{ha}+$ $2 \% \mathrm{~K}_{2} \mathrm{O}$ and $57 \mathrm{Kg} \mathrm{K}_{2} \mathrm{O} /$ ha with insignificant difference as compared with other potassium treatments. Also, a significant increase was recorded in $\mathrm{Na}^{+}$content in leaves under control $\mathrm{K}^{+}$treatment $\left(0 \mathrm{~K}_{2} \mathrm{O} / \mathrm{ha}\right)$.

Linear relationship was found between potassium fertilization rates (soil dressing, foliar application as well as $28.6 \mathrm{KgK}_{2} \mathrm{O} / \mathrm{ha}+$ foliar1 and $2 \%$ applications) and fresh forage weight $\mathrm{t} / \mathrm{ha}$. The linear regression equation showed that as potassium fertilizer rate (soil dressing) increased by one unite/ha, fresh forage weight/ha increased by $1.139 \mathrm{t} / \mathrm{ha}$ and as potassium fertilizer rate (foliar application) increased by one unite/ha, fresh forage weight/ha increased by $3.112 \mathrm{t} / \mathrm{ha}$. In addition, the highest fresh forage weight/ha (125.850 t/ha) was produced under treatment of $28.6 \mathrm{KgK}_{2} \mathrm{O} / \mathrm{ha}+2 \% \mathrm{~K}_{2} \mathrm{O}$.

Highly positive significant correlation coefficients were found for all traits except the relation between $\mathrm{Na}^{+}$content and other traits which was a highly significant negative relationship.

Profitability assessment results revealed that addition of $28.6 \mathrm{KgK}_{2} \mathrm{O} /$ ha with foliar application of $2 \% \mathrm{~K}_{2} \mathrm{O}$ as potassin $30 \% \mathrm{~K}_{2} \mathrm{O}$ followed by addition of $57.14 \mathrm{KgK}_{2} \mathrm{O} /$ ha markedly obtained the highest values of alfalfa yield with insignificant difference. These results were incorporated with the highest values of output, net income and the investment ratio.

Results indicated that controlling soil fertility, especially $\mathrm{K}^{+}$as soil application, in addition to foliar application under saline calcareous soil condition is considered to be one of the most important factors to overcome the negative effects of salinity stress.
\end{abstract}

Keywords: alfalfa, saline soil, potassium foliar and/or soil application.

\section{INTRODUCTION}

Alfalfa (Medicago sativa L.) is a permanent forage legume in the newly reclaimed area in Egypt. It is an important forage source for all cases of livestock because of its wide adaptability, high yield, good quality (digestibility and protein content), resistance to frequent cuttings, (Goplen et $a l .$, 1982) and seasonal distribution through the year (Castroluna 2009). Moreover, it is often used to improve soil fertility due to its capacity of symbiotic nitrogen fixation. Alfalfa is moderately salinetolerant legume and can withstand an equivalent of $20 \mathrm{mM}$ sodium chloride (Bertrand et al., 2015)

Soil salinity is one of the most influential abiotic stresses that affected crop growth and limits agricultural production worldwide and is becoming a global issue of land degradation, with more prevalence in arid and semi-arid regions, (Tanji, 1990). Increased salinization of arable land is expected to have devastating global effect, resulting in $30 \%$ land loss within the next 25 years and up to $50 \%$ by the middle of 21 st Century (Wang et al., 2003).

Salt stress causes many adverse effects on growth, development, yield and its quality (Ashraf and Harris, 2004) due to a high osmotic potential of soil solution (osmotic stress), specific ion effects (ion stress) and reactive oxygen species production (ROS) (oxidative stress) (Flowers et al., 1977, and Greenway and Munns 1980) which resulting damage of lipids membrane, protein, enzymes, pigments and nucleic acid (Gill and Tuteja 2010 and Chawla et al. 2013). In addition, the presence of high root zone salt concentration causes higher osmotic pressure in soil solution than in plant cells, 
reducing the ability of plants to uptake water and essential minerals like potassium and calcium, (Munns et al., 2006). In severe salt stress, the soil solution becomes hyper-osmotic, causing the root cell to lose water, which results in severe wilting or plant senescence, (Munns, 2002) Sodium ions absorbed by plant roots can be harmful to the plant (Tuteja, 2007). Since sodium and potassium ions are both monovalent cations, they compete for uptake by the plant under fully hydrated saline conditions, (Schachtman and Liu, 1999) resulting in a deficiency in potassium, an essential macronutrient required for normal metabolic functions. Increased concentration of sodium and chloride ions in the cytoplasm can disrupt cellular processes, causing damage to photosynthetic apparatus as well as cells dehydration (Munns and Tester, 2008 and Ashraf and Harris, 2013). Therefore, limiting excess amount of sodium in cytosol and increasing the cellular potassium to sodium ratio are crucial for salt tolerance, (Annunziata et al., 2017 and Carillo et al. 2019). This means that maintenance of regular photosynthetic rate and stable $\mathrm{K}^{+} / \mathrm{Na}^{+}$ratio are important traits for salt- tolerant alfalfa cultivars, (Bhattarai et al., 2020). Eman et al. (2009), studied the effect of salt stress on two alfalfa genotypes and found that dry matter production decreased under high salinity level in both cultivars.

Potassium is a macronutrient that plays essential roles related to the osmotic adjustment, to maintaining turgor and to regulating the membrane potential, cytoplasmic homeostasis, protein synthesis, and enzyme activation under salt stress, (Almeida et al., 2017). Also, maintaining the cellular $\mathrm{K}^{+}$content above a certain threshold and maintaining a low $\mathrm{K}^{+} / \mathrm{Na}^{+}$ratio is crucial for plant growth and salt tolerance, Thus, higher application of $\mathrm{K}^{+}$increase the $\mathrm{K}^{+}$content in plant cell and reduce the $\mathrm{Na}^{+}$concentration, which increase the $\mathrm{K}^{+} / \mathrm{Na}^{+}$ratio. The HTK (high- affinity $\mathrm{K}^{+}$ transporter) mediates $\mathrm{Na}^{+}$- specific transport or $\mathrm{K}^{+}$$\mathrm{Na}^{+}$co-transport, which have vital roles in plant $\mathrm{Na}^{+}$ tolerance, ( Su et al., a 2015 and Su et al., b 2015). However, exogenous $\mathrm{K}^{+}$can positively correlate with plant root and shoot growth during salinity stress and the $\mathrm{K}^{+}$deficient stage. El-Sharkawy et al., (2017) studied the effect of various rate of potassium sulfate $\left(\mathrm{K}_{2} \mathrm{SO}_{4}\right)$ nanoparticles on alfalfa growth and physiological response under salt stress. They found that adding $\mathrm{K}_{2} \mathrm{SO}_{4}$ nanoparticles at the rate $1 / 8$ level resulted in the highest shoot dry weight, relative yield, root length and root dry weight and also, enhanced the plant's physiological response to salt stress by reducing electrolyte leakage, increasing catalase, proline content and antioxidant enzymes activity. In addition, they suggested that the different rate of $\mathrm{K}_{2} \mathrm{SO}_{4}$ nanoparticles affected significantly $\mathrm{Na} / \mathrm{K}$ ratio and concentrations of $\mathrm{Ca}, \mathrm{P}, \mathrm{Cu}, \mathrm{Mn}$ and $\mathrm{Zn}$ in plant tissue.

Excessive application of potassium fertilization as a soil dressing may raise toxicity and environmental pollution as well as increasing $\mathrm{K}^{+}$ fertilization costs. Thus, foliar spray of potassium fertilizer as a supplementary fertilization is an active way to increase absorption of $\mathrm{K}^{+}$and other nutrients, in addition to enhance the $\mathrm{K}^{+}$use efficiency and reduce potassium fertilizer costs.

The present work aimed to mitigate the negative effects of salinity stress by potassium fertilization as a soil dressing and/or foliar application as well as in combination on alfalfa plants grown under saline calcareous soil conditions at Nubaria region. Also, to study the relationship between potassium fertilizer rates and alfalfa forage yields through regression analysis and the correlations between different alfalfa traits.

\section{MATERIALS AND METHODS}

A field experiment was conducted along the period duration beginning in September 2015 to November 2017 at the experimental farm of Nubaria Agricultural Research station, El-Behira Governorate, Egypt, that represent newly reclaimed saline calcareous soil. The aim of the present work was to evaluate the physiological response, forage productivity and quality of local alfalfa cultivar (Nubaria-1) as affected by different foliar and soil applications of potassium fertilization treatments under saline calcareous soil conditions at Nubaria region.

Representative initial soil samples at a depth of 0-40 cm. were collected before application of the experimental treatments, soil preparation and planting and at the end of each year for analysis according to Chapman and Pratt (1978) and Black (1965). Soil physical, chemical and fertility properties of the investigated site were shown in Table (1). The surface soil layer $(0-40 \mathrm{~cm})$ has light texture of sandy loam with a high content of $\mathrm{CaCO}_{3}$ $\%$ (Average value $23.6 \%$ ) with a bulk density value $1.37 \mathrm{~g} / \mathrm{cm}^{3}$ and available water $28.73 \%$. The average soil $\mathrm{pH}$ and $\mathrm{EC}(\mathrm{dS} / \mathrm{m})$ values were 8.37 and 6.85 , respectively, which indicate that soil was saline with an alkaline reaction. The available macro and micronutrient concentrations were very low and below the critical limits.

Climatic data at Nubaria region mainly air and soil temperature and relative humidity $\%$ were recorded during the experimental duration of the two years through winter, spring, summer and autumn (Table 2).

The experiment was carried out in a randomized complete block design with four replicates. 
Table 1: Initial Soil physicochemical characteristics and fertility status of the experimental sit.

\begin{tabular}{|c|c|c|c|}
\hline Soil characteristics & $\begin{array}{l}\text { Mean value } \\
\text { Sep. } 2015\end{array}$ & $\begin{array}{l}\text { Mean value Sep. } \\
2016\end{array}$ & $\begin{array}{l}\text { Mean value } \\
\text { Nov. } 2017\end{array}$ \\
\hline Soil pH $(1: 2.5)$ & 8.37 & 8.30 & 8.29 \\
\hline Soil EC $(\mathrm{dS} / \mathrm{m})^{*}$ & 6.85 & 5.73 & 4.95 \\
\hline Total $\mathrm{CaCO}_{3} \%$ & 23.6 & 23.46 & 23.59 \\
\hline Soil bulk density $\left(\mathrm{g} / \mathrm{cm}^{3}\right)$ & 1.37 & 1.33 & 1.34 \\
\hline Soil Texture & Sandy loom & Sandy loom & Sandy loom \\
\hline Available water\% & 28.73 & 28.83 & 29.11 \\
\hline \multicolumn{4}{|l|}{ Soluble Cations (meq/L) } \\
\hline $\mathrm{Ca}^{2+}$ & 19.86 & 18.64 & 14.22 \\
\hline $\mathrm{Mg}^{2+}$ & 6.93 & 3.01 & 1.98 \\
\hline $\mathrm{Na}^{+}$ & 36.74 & 32.11 & 29.94 \\
\hline $\mathrm{K}^{+}$ & 4.97 & 3.72 & 3.36 \\
\hline \multicolumn{4}{|l|}{ Soluble anions $(\mathrm{meq} / \mathrm{L})$} \\
\hline $\mathrm{CO}_{3}{ }^{2-}$ & ------- & ------- & -------- \\
\hline $\mathrm{HCO}_{3}^{-}$ & 8.94 & 7.62 & 6.31 \\
\hline $\mathrm{Cl}^{-}$ & 40.17 & 36.11 & 31.42 \\
\hline $\mathrm{SO}_{4}^{2-}$ & 19.39 & 13.57 & 11.77 \\
\hline $\mathrm{O} . \mathrm{M}(\%)$ & 0.21 & 0.27 & 0.31 \\
\hline \multicolumn{4}{|c|}{ Available macronutrients(ppm) } \\
\hline $\mathrm{N}$ & 37.11 & 37.43 & 38.07 \\
\hline $\mathrm{P}$ & 3.74 & 3.52 & 3.69 \\
\hline K & 82.93 & 91.41 & 93.20 \\
\hline \multicolumn{4}{|c|}{ DTPA-extractable micronutrients(ppm) } \\
\hline $\mathrm{Zn}$ & 1.39 & 1.27 & 1.31 \\
\hline $\mathrm{Fe}$ & 2.18 & 2.26 & 2.24 \\
\hline $\mathrm{Mn}$ & 0.97 & 0.92 & 0.88 \\
\hline
\end{tabular}

Where*: soil peat.

Table 2: Number, date of cuts and Climatic data cutting time at the experimental site.

\begin{tabular}{|c|c|c|c|c|c|c|}
\hline Year & Season & $\begin{array}{l}\text { No.of } \\
\text { cut }\end{array}$ & Date of cut & $\begin{array}{c}\text { Air } \\
\text { temperature }\end{array}$ & $\begin{array}{c}\text { Soil } \\
\text { temperature }\end{array}$ & $\begin{array}{c}\text { Relative } \\
\text { humidity \% }\end{array}$ \\
\hline \multirow{9}{*}{$\begin{array}{l}2015 / 2016 \\
\text { (First year) }\end{array}$} & Winter & 1 & $18 / 12 / 2015$ & 19.7 & 11.5 & 66.1 \\
\hline & & 2 & $3 / 2 / 2016$ & 13.1 & 9.4 & 68.7 \\
\hline & Spring & 1 & $20 / 3 / 2016$ & 20.8 & 10.3 & 53.4 \\
\hline & & 2 & $14 / 5 / 2016$ & 24.4 & 14.6 & 47.8 \\
\hline & Summer & 1 & $15 / 6 / 2016$ & 28.9 & 17.8 & 50.9 \\
\hline & & 2 & $16 / 7 / 2016$ & 34.3 & 22.4 & 59.3 \\
\hline & & 3 & $14 / 8 / 2016$ & 35.6 & 28.1 & 61.5 \\
\hline & Autumn & 1 & $15 / 9 / 2016$ & 32.4 & 20.6 & 51.2 \\
\hline & & 2 & $2 / 11 / 2016$ & 23.5 & 19.8 & 71.1 \\
\hline \multirow{9}{*}{$\begin{array}{l}2016 / 2017 \\
\text { (Second year) }\end{array}$} & Winter & 1 & $19 / 12 / 2016$ & 16.8 & 10.8 & 61.9 \\
\hline & & 2 & $5 / 2 / 2017$ & 14.3 & 7.6 & 67.8 \\
\hline & Spring & 1 & $19 / 3 / 2017$ & 18.9 & 11.3 & 70.7 \\
\hline & & 2 & 4/5/2017 & 25.6 & 17.4 & 64.3 \\
\hline & Summer & 1 & 6/6/2017 & 33.1 & 20.9 & 50.1 \\
\hline & & 2 & $8 / 7 / 2017$ & 35.2 & 23.4 & 46.4 \\
\hline & & 3 & $10 / 8 / 2017$ & 34.9 & 24.5 & $\begin{array}{l}51.8 \\
\end{array}$ \\
\hline & Autumn & 1 & $13 / 9 / 2017$ & 31.4 & 20.5 & 57.7 \\
\hline & & 2 & $2 / 11 / 2017$ & 24.6 & 15.4 & 73.6 \\
\hline
\end{tabular}


The experimental plot area was $6 \mathrm{~m}^{2}(2 \times 3 \mathrm{~m})$ and consisted of 10 rows, $20 \mathrm{~cm}$ apart and $3 \mathrm{~m}$ long, using Nubaria1 alfalfa seeds (Obtained From Forage Corps Department, Field Corps Research Institute, ARC, Egypt)

Alfalfa inoculated seeds by Rizobium meliloti at the rate of $48 \mathrm{Kg} \mathrm{ha}^{-1}$ drilling in the beginning of September 2015. Calcium superphosphate $\left(15.5 \% \mathrm{P}_{2} \mathrm{O}_{2}\right)$ was applied at the rate of $148 \mathrm{Kg} \mathrm{P}_{2} \mathrm{O}_{5}$ $\mathrm{ha}^{-1}$ during land preparation and nitrogen fertilizer in the form of ammonium nitrate $(33.5 \% \mathrm{~N})$ at the rate of $47.6 \mathrm{Kg} \mathrm{N} /$ ha was added in two equal doses after 21 and 42 days from planting for the first year and after the $9^{\text {th }}$ and $10^{\text {th }}$ cuts for the second year. Potassium fertilization treatments were: K1; Control (without potassium fertilizer), $\mathrm{K} 2 ; 57.14 \mathrm{Kg} \mathrm{K}_{2} \mathrm{O}$ / ha as potassium sulphate $\left(48 \% \mathrm{~K}_{2} \mathrm{O}\right), \mathrm{K} 3 ; 28.6 \mathrm{Kg} \mathrm{K}_{2} \mathrm{O}$

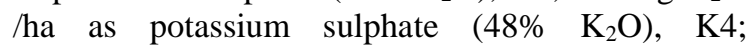
Potassium foliar application (1\% as potassin $30 \%$ $\mathrm{K}_{2} \mathrm{O}$ ), K5; Potassium foliar application (2\% as Potassin 30\% $\mathrm{K}_{2} \mathrm{O}$ ), K6; $28.6 \quad \mathrm{Kg} \quad \mathrm{K}_{2} \mathrm{O}$ /ha + potassium foliar application (1\% as Potassin 30\% $\mathrm{K}_{2} \mathrm{O}$ ), $\mathrm{K} 7 ; 28.6 \mathrm{Kg} \mathrm{K}_{2} \mathrm{O} / \mathrm{ha}+$ potassium foliar application (2\% as Potassin $\left.30 \% \quad \mathrm{~K}_{2} \mathrm{O}\right)$. Soil application of potassium fertilizer treatments in the form of potassium sulphate $\left(48 \% \mathrm{~K}_{2} \mathrm{O}\right)$ were applied on two equal doses with $\mathrm{N}$ fertilizer application in the two experimental years. Potassium foliar treatments were sprayed after 65 days from sowing besides 15 days before each cut. All other agricultural practices (Irrigation, weed control ...etc.) were followed as recommended at the site.

Nine cuts/year were harvested from alfalfa, with a total of 18 cuts during the experimental period, the first cut was taken after 80 days from sowing and followed every 45 days in winter, spring and autumn seasons and every 30 days in summer season.

Ten representative plants were collected randomly from each plot before cutting to determine some growth parameters including plant height (cm.) and leaf/stem ratio. Leaves of alfalfa plant samples were separated from stems then leaves and stem samples were oven dried at $70 \mathrm{C}^{\circ}$ for 72 hours till constant weight, then the dry separated leaves and stems were weighed and the leaf/ stem ratio (L.S.R) was calculated for each treatment.

Alfalfa forage yield (t/ha) was measured by harvesting each plot $\left(6.0 \mathrm{~m}^{2}\right)$ and the forage fresh weight was recorded in the field $\left(\mathrm{Kg} / 6 \mathrm{~m}^{2}\right)$ and subsamples were collected (fresh forage of about $200 \mathrm{~g}$ ) weighed exactly and then sent to the lab for oven drying and reweighed to determine the dry matter\% and forage dry weight. Fresh and dry forage yields for each cut and each plot were accumulated to calculate the total fresh and dry forage yield $(\mathrm{t} / \mathrm{ha})$ for each season during the two experimental years.
To determine forage quality parameters, 3 macronutrients $\left(\mathrm{N}, \mathrm{K}^{+}\right.$and $\left.\mathrm{Na}^{+}\right)$and protein content were determined according to A.O.A.C. (1990). Crude protein was determined using Automatic Kjeldahel instruments to determine $\mathrm{N}$ content. Then protein content was calculated by multiplying total nitrogen percentage by factor of 6.25 (A.O.A.C., 1990).

At each cut, leaf samples were immediately weighed (fresh weight, $(\mathrm{FW})$ ) and transferred into sealed flasks, then rehydrated in water for $5 \mathrm{~h}$ until fully turgid, surface swabbed and reweighed (turgid weight, (TW)). Leaf samples were oven dried at 70 $\mathrm{C}^{\circ}$ for $48 \mathrm{~h}$ and reweighed (dry weight, (DW)). Relative water content (RWC, \%) of leaves was calculated according to Lazcano-Ferrat and Lovatt (1999) as follows:

\section{RWC $=(($ FW - DW $) /($ TW - DW $)) *(\mathbf{1 0 0})$}

Profitability calculations for the input and output values for different experimental treatments under study were done to determine the total net income and the investment ratio (I.R) for all tested treatments.

Collected data were statistically analyzed according to procedures outlined by Snedecor and Cochran (1980) using SAS software (2014). The least significant differences (L.S.D.) at 0.05 were calculated to separate the mean values. The regression analysis were done according to Montgomery and Peck (1982) and correlation analysis according to Steel and Torrie (1980).

\section{RESULTS AND DISCUSSION}

\section{Growth traits:}

Data presented in Tables (3, 4, 5, and 6) show the effect of potassium fertilizer rates on two growth traits of alfalfa including plant height and leaf stem ratio (L.S.R) in each cut. It is worthy to mention that summer season,cuts gave the taller plants and the highest leaf/stem ratio followed by spring, autumn and winter seasons in descending order in both years. This result could be due to the change in temperature and its effect on the elongation of internodes, (Mousa et al., 1996).

According to the data in Tables(3, 4, 5, and 6), significant differences were observed due to potassium application in plant height and L.S.R for the individual cuts in the first and second years except the $1^{\text {st }}$ and $9^{\text {th }}$ cuts in the first year and the $2^{\text {nd }}$ cut in the second year for plant height.

Application of potassium fertilizer significantly enhanced plant height and L.S.R under saline soil conditions compared to the untreated plants. The highest values of plant height were obtained from adding $28.6 \mathrm{~kg} \mathrm{~K} 2 \mathrm{O}+2 \% \mathrm{~K}_{2} \mathrm{O}$ followed by adding $57.14 \mathrm{Kg} \mathrm{K} 2 \mathrm{O}$ treatments with insignificant difference in $1^{\text {st }}, 2^{\text {nd }}, 3^{\text {rd }}, 4^{\text {th }}, 5^{\text {th }}$ and $6^{\text {th }}$ cutes in the first year and $1^{\text {st }}, 2^{\text {nd }}, 3^{\text {rd }}, 4^{\text {th }}, 5^{\text {th }}$ and $9^{\text {th }}$ in the second year. While the highest values for plant height in $7^{\text {th }}$ 
and $8^{\text {th }}$ cuts in the first year and $6^{\text {th }}, 7^{\text {th }}$ and $8^{\text {th }}$ in the second year were obtained from adding $28.6 \mathrm{~kg} \mathrm{~K}_{2} \mathrm{O}$ $+2 \% \mathrm{~K}_{2} \mathrm{O}$ followed by $57.14 \mathrm{~kg} \mathrm{~K}_{2} \mathrm{O}$ and $28.6 \mathrm{~kg}$ $\mathrm{K}_{2} \mathrm{O}+1 \% \quad \mathrm{~K}_{2} \mathrm{O}$ with insignificant difference. However, the highest L.S.R values were obtained from adding $28.6 \mathrm{~kg} \mathrm{~K}_{2} \mathrm{O}+2 \% \mathrm{~K}_{2} \mathrm{O}$ followed by $57.14 \mathrm{~kg} \mathrm{~K}_{2} \mathrm{O}$ with insignificant difference in all cuts in the first season except $5^{\text {th }}, 6^{\text {th }}$ and $8^{\text {th }}$ cut and in the second season except $7^{\text {th }}$ cut. The highest values for L.S.R in the $5^{\text {th }}, 6^{\text {th }}$ and $8^{\text {th }}$ cuts in the first season and $7^{\text {th }}$ cutting in the second season were obtained from plants received $28.6 \mathrm{~kg} \mathrm{~K}_{2} \mathrm{O}+2 \%$
$\mathrm{K}_{2} \mathrm{O}$ followed by $57.14 \mathrm{~kg} \mathrm{~K}_{2} \mathrm{O}$ and $28.6 \mathrm{~kg} \mathrm{~K}_{2} \mathrm{O}$ $+1 \% \quad \mathrm{~K}_{2} \mathrm{O}$ with insignificant differences. These findings are due to that potassium is essential for the function and performance of many plant enzymes; at least 60 enzymes require $\mathrm{K}^{+}$as a cofactor for activation (Hawkesford et al., 2012). In addition, Zizy and Awad (2018), suggested that increasing the amount of $\mathrm{K}^{+}$by spraying plants directly had a positive effect on plant height, loading and transport of nutrients in mono-cut Egyptian clover under saline soil.

Table 3: Effect of Potassium fertilizer treatments on plant height (cm) for 9 cuts in 2015/2016 season.

\begin{tabular}{|c|c|c|c|c|c|c|c|c|c|c|}
\hline \multirow{2}{*}{$\begin{array}{l}\text { Potassium } \\
\text { fertilizer } \\
\text { treatments }\end{array}$} & \multicolumn{2}{|c|}{ Winter } & \multicolumn{2}{|c|}{ Spring } & \multicolumn{3}{|c|}{ Summer } & \multicolumn{2}{|c|}{ Autumn } & \multirow[b]{2}{*}{$\begin{array}{l}\text { Means } \\
\text { of Cut }\end{array}$} \\
\hline & $\begin{array}{c}\mathbf{1}^{\text {st }} \\
\text { Cut }\end{array}$ & $\begin{array}{c}2^{\text {nd }} \\
\text { Cut }\end{array}$ & $\begin{array}{c}\mathbf{3}^{\text {rd }} \\
\text { Cut }\end{array}$ & $\begin{array}{c}4^{\text {th }} \\
\text { Cut }\end{array}$ & $\begin{array}{c}5^{\text {th }} \\
\text { Cut }\end{array}$ & $\begin{array}{c}\mathbf{6}^{\text {th }} \\
\text { Cut }\end{array}$ & $\begin{array}{c}7^{\text {th }} \\
\text { Cut }\end{array}$ & $\begin{array}{c}\mathbf{8}^{\text {th }} \\
\text { Cut }\end{array}$ & $\begin{array}{c}9^{\text {th }} \\
\text { Cut }\end{array}$ & \\
\hline K1 & 43.73 & 43.17 & 42.66 & 44.36 & 49.40 & 50.16 & 45.43 & 45.54 & 45.41 & 45.54 \\
\hline $\mathrm{K} 2$ & 48.73 & 51.59 & 51.23 & 54.24 & 57.67 & 58.87 & 63.10 & 55.66 & 49.21 & 54.47 \\
\hline K3 & 48.40 & 47.24 & 48.67 & 51.81 & 54.56 & 57.14 & 59.35 & 52.30 & 47.81 & 51.92 \\
\hline K4 & 45.67 & 43.94 & 46.33 & 47.61 & 53.16 & 52.94 & 58.64 & 47.79 & 46.26 & 49.14 \\
\hline K5 & 47.13 & 45.02 & 47.30 & 50.87 & 54.01 & 55.80 & 58.64 & 49.59 & 47.45 & 50.64 \\
\hline K6 & 48.62 & 48.13 & 49.13 & 52.98 & 56.12 & 57.40 & 61.14 & 55.21 & 48.42 & 53.01 \\
\hline K7 & 50.17 & 51.85 & 51.41 & 55.26 & 58.47 & 60.10 & 63.57 & 55.81 & 50.61 & 55.25 \\
\hline LSD $_{0.05}$ & NS & 2.72 & 2.13 & 1.20 & 1.53 & 1.44 & 2.01 & 1.49 & $\mathrm{NS}$ & \\
\hline
\end{tabular}

Table 4: Effect of Potassium fertilizer treatments on plant height $(\mathrm{cm})$ for 9 cuts in 2016/2017 season.

\begin{tabular}{|c|c|c|c|c|c|c|c|c|c|c|c|}
\hline \multirow{2}{*}{$\begin{array}{l}\text { Potassium } \\
\text { treatments }\end{array}$} & \multirow[t]{2}{*}{ fertilizer } & \multicolumn{2}{|c|}{ Winter } & \multicolumn{2}{|c|}{ Spring } & \multicolumn{3}{|c|}{ Summer } & \multicolumn{2}{|c|}{ Autumn } & \multirow[b]{2}{*}{$\begin{array}{l}\text { Means } \\
\text { of Cut }\end{array}$} \\
\hline & & $\begin{array}{c}1^{\text {st }} \\
\text { Cut }\end{array}$ & $\begin{array}{l}2^{\text {nd }} \\
\text { Cut }\end{array}$ & $\begin{array}{l}3^{\text {rd }} \\
\text { Cut }\end{array}$ & $\begin{array}{l}4^{\text {th }} \\
\text { Cut }\end{array}$ & $\begin{array}{c}5^{\text {th }} \\
\text { Cut }\end{array}$ & $\begin{array}{c}\mathbf{6}^{\text {th }} \\
\text { Cut }\end{array}$ & $\begin{array}{c}7^{\text {th }} \\
\text { Cut }\end{array}$ & $\begin{array}{l}8^{\text {th }} \\
\text { Cut }\end{array}$ & $\begin{array}{c}9^{\text {th }} \\
\text { Cut }\end{array}$ & \\
\hline K1 & & 47.31 & 46.51 & 49.11 & 53.09 & 52.74 & 55.26 & 57.94 & 50.46 & 46.26 & 50.96 \\
\hline $\mathrm{K} 2$ & & 56.51 & 54.86 & 56.21 & 66.00 & 62.64 & 64.93 & 67.55 & 55.66 & 53.93 & 59.81 \\
\hline K3 & & 51.99 & 53.44 & 53.74 & 57.88 & 60.81 & 62.52 & 65.91 & 53.45 & 52.53 & 56.91 \\
\hline $\mathrm{K} 4$ & & 49.04 & 50.68 & 51.44 & 54.60 & 59.15 & 59.71 & 61.26 & 51.34 & 51.10 & 54.25 \\
\hline K5 & & 50.11 & 52.78 & 52.34 & 55.89 & 59.65 & 60.10 & 65.85 & 52.51 & 51.96 & 55.68 \\
\hline K6 & & 54.20 & 53.48 & 54.21 & 64.65 & 61.41 & 64.64 & 67.40 & 53.50 & 52.58 & 58.45 \\
\hline K7 & & 56.86 & 55.38 & 57.04 & 66.50 & 63.29 & 65.33 & 69.14 & 54.29 & 55.75 & 60.39 \\
\hline $\operatorname{LSD}_{0.05}$ & & 2.81 & NS & 1.92 & 1.30 & 1.20 & 2.08 & 1.42 & 2.23 & 1.31 & \\
\hline
\end{tabular}

Table 5: Effect of Potassium fertilizer treatments on leaf stem ratio (LSR) for 9 cuts in 2015/2016 season.

\begin{tabular}{|c|c|c|c|c|c|c|c|c|c|c|}
\hline \multirow{2}{*}{$\begin{array}{l}\text { Potassium } \\
\text { fertilizer } \\
\text { treatments }\end{array}$} & \multicolumn{2}{|c|}{ Winter } & \multicolumn{2}{|c|}{ Spring } & \multicolumn{3}{|c|}{ Summer } & \multicolumn{2}{|c|}{ Autumn } & \multirow[b]{2}{*}{$\begin{array}{l}\text { - Means } \\
\text { of Cut }\end{array}$} \\
\hline & $\begin{array}{c}1^{\text {st }} \\
\text { Cut }\end{array}$ & $\begin{array}{c}2^{\text {nd }} \\
\text { Cut }\end{array}$ & $\begin{array}{c}3^{\text {rd }} \\
\text { Cut }\end{array}$ & $\begin{array}{c}4^{\text {th }} \\
\text { Cut }\end{array}$ & $\begin{array}{c}5^{\text {th }} \\
\text { Cut }\end{array}$ & $\begin{array}{c}\text { 6 }^{\text {th }} \\
\text { Cut }\end{array}$ & $\begin{array}{c}7^{\text {th }} \\
\text { Cut }\end{array}$ & $\begin{array}{c}8^{\text {th }} \\
\text { Cut }\end{array}$ & $\begin{array}{c}9^{\text {th }} \\
\text { Cut }\end{array}$ & \\
\hline K1 & 37.51 & 39.60 & 43.58 & 46.33 & 48.32 & 48.11 & 49.31 & 42.04 & 41.35 & 44.01 \\
\hline $\mathrm{K} 2$ & 45.55 & 49.35 & 54.63 & 61.28 & 59.64 & 59.32 & 58.63 & 50.38 & 49.84 & 54.25 \\
\hline K3 & 41.05 & 47.31 & 50.21 & 56.84 & 55.32 & 55.61 & 54.21 & 46.05 & 46.58 & 50.35 \\
\hline K4 & 39.62 & 45.23 & 45.62 & 50.31 & 52.14 & 52.64 & 51.92 & 44.15 & 44.21 & 47.31 \\
\hline K5 & 40.21 & 46.95 & 46.28 & 53.26 & 54.35 & 54.92 & 53.27 & 45.68 & 45.60 & 48.94 \\
\hline K6 & 43.52 & 48.04 & 52.61 & 59.25 & 58.47 & 57.34 & 56.31 & 48.72 & 47.28 & 52.56 \\
\hline K7 & 46.83 & 49.47 & 55.09 & 62.13 & 61.25 & 60.35 & 60.59 & 50.19 & 49.51 & 55.04 \\
\hline $\operatorname{LSD}_{0.05}$ & 1.24 & 1.29 & 2.10 & 1.92 & 1.84 & 2.04 & 1.99 & 1.56 & 2.19 & \\
\hline
\end{tabular}


Table 6: Effect of Potassium fertilizer treatments on leaf stem ratio (LSR) for 9 cuts in 2016/2017 season.

\begin{tabular}{|c|c|c|c|c|c|c|c|c|c|c|}
\hline \multirow{2}{*}{$\begin{array}{l}\text { Potassium } \\
\text { fertilizer } \\
\text { treatments }\end{array}$} & \multicolumn{2}{|c|}{ Winter } & \multicolumn{2}{|c|}{ Spring } & \multicolumn{3}{|c|}{ Summer } & \multicolumn{2}{|c|}{ Autumn } & \multirow[b]{2}{*}{$\begin{array}{l}\text {-Means } \\
\text { of Cut }\end{array}$} \\
\hline & $\begin{array}{l}1^{\text {st }} \\
\text { Cut }\end{array}$ & $\begin{array}{l}2^{\text {nd }} \\
\text { Cut }\end{array}$ & $\begin{array}{l}3^{\text {rd }} \\
\text { Cut }\end{array}$ & $\begin{array}{l}4^{\text {th }} \\
\text { Cut }\end{array}$ & $\begin{array}{l}5^{\text {th }} \\
\text { Cut }\end{array}$ & $\begin{array}{l}6^{\text {th }} \\
\text { Cut }\end{array}$ & $\begin{array}{l}7^{7^{\text {th }}} \\
\text { Cut }\end{array}$ & $\begin{array}{l}8^{\text {th }} \\
\text { Cut }\end{array}$ & $\begin{array}{l}9^{\text {th }} \\
\text { Cut }\end{array}$ & \\
\hline $\mathrm{K} 1$ & 40.36 & 41.33 & 45.29 & 47.12 & 49.09 & 49.27 & 48.66 & 46.91 & 43.41 & 45.71 \\
\hline $\mathrm{K} 2$ & 47.58 & 51.38 & 55.46 & 59.43 & 59.78 & 60.35 & 58.73 & 53.83 & 51.11 & 55.29 \\
\hline $\mathrm{K} 3$ & 45.24 & 48.05 & 53.93 & 54.51 & 55.56 & 54.81 & 55.21 & 50.76 & 47.35 & 51.71 \\
\hline K4 & 42.32 & 44.12 & 50.03 & 53.62 & 53.37 & 52.56 & 51.02 & 48.82 & 45.45 & 49.03 \\
\hline $\mathrm{K} 5$ & 43.87 & 45.68 & 50.87 & 54.41 & 55.14 & 54.44 & 54.70 & 49.41 & 46.61 & 50.57 \\
\hline K6 & 46.13 & 49.75 & 54.07 & 57.70 & 57.91 & 56.65 & 57.64 & 51.31 & 49.47 & 53.40 \\
\hline K7 & 48.06 & 51.56 & 56.38 & 60.37 & 61.55 & 62.73 & 62.25 & 53.62 & 51.05 & 56.39 \\
\hline $\operatorname{LSD}_{0.05}$ & 1.42 & 1.61 & 1.36 & 1.84 & 1.79 & 2.08 & 1.96 & 2.34 & 1.60 & \\
\hline
\end{tabular}

\section{Alfalfa forage Yield:}

Results in Tables $(7,8,9,10,11$ and12) revealed that fresh and dry forage yields as well as dry matter were significantly influenced by potassium fertilization as compared to control (0-potassium) in all cuts in the both years except the $2^{\text {nd }}$ and $7^{\text {th }}$ cuts for dry matter in both years. It can be noticed that the fresh and dry forage yields of winter and autumn growth were apparently lower than that of spring and summer. Such results is mainly due to the fact that photoperiod and soil temperature both affect growth rate, stem initiation, and allocation of photosynthetic products to the development of roots and stem (Mueller and Teuber, 2007). The warmer temperatures and longer days cause more rapid plant development and grater cell wall lignification than occurs in cooler temperatures (Undersander et al., 2011). Results showed that the highest values were obtained from plants received $28.6 \mathrm{KgK}_{2} \mathrm{O} / \mathrm{ha}+$ $2 \% \mathrm{~K}_{2} \mathrm{O}$ and plants received $57.14 \mathrm{Kg} \mathrm{K}_{2} \mathrm{O} /$ ha with insignificant difference for fresh forage in $1^{\text {st }}, 2^{\text {nd }}$, $3^{\text {rd }}, 4^{\text {th }}, 8^{\text {th }}$, and $9^{\text {th }}$ cuts in first year and in $2^{\text {nd }}, 3^{\text {rd }}$, $4^{\text {th }}, 5^{\text {th }}$, cuts in second year and for dry forage in all cuts except $6^{\text {th }}$ and $7^{\text {th }}$ in first year and in 3 rd, $4^{\text {th }}, 5^{\text {th }}$ and $6^{\text {th }}$ cuts in the second year, and for dry matter in $5^{\text {th }}$ and $6^{\text {th }}$ cuts in the first year and $3^{\text {rd }}, 4^{\text {th }}$ and $5^{\text {th }}$ cuts in the second year. However, the highest values of fresh forage (in $1^{\text {st }}, 3^{\text {rd }}, 8^{\text {th }}$ and $9^{\text {th }}$ for first year and in $1^{\text {st }}, 6^{\text {th }}$ and $9^{\text {th }}$ for second year) and dry forage in $\left(6^{\text {th }}\right.$ and $\left.7^{\text {th }}\right)$ for the first year and in $\left(7^{\text {th }}, 8^{\text {th }}\right.$ and $9^{\text {th }}$ ) for the second year, were obtained from plants received $28.6 \mathrm{Kg} \quad \mathrm{K}_{2} \mathrm{O}+2 \% \mathrm{~K}_{2} \mathrm{O}$, plants received $57.14 \mathrm{KgK}_{2} \mathrm{O} / \mathrm{ha}$ and plants received $28.6 \mathrm{KgK}_{2} \mathrm{O}+$ $1 \% \mathrm{~K}_{2} \mathrm{O}$ with insignificant differences. The highest dry forage yield in $1^{\text {st }}$ cut in the first year was achieved when plants received $28.6 \mathrm{Kg} \mathrm{K}_{2} \mathrm{O} / \mathrm{ha}+$ $2 \% \mathrm{~K}_{2} \mathrm{O}$. Such finding was attributed to the role of potassium in dry matter accumulation. Moreover, potassium fertilizer mitigates the adverse effect of salinity and the increase of values of growth due to the basal and foliar application of $(\mathrm{K})$ might have an important role in photosynthesis and its possible role in plant metabolism involved activation of many enzymes. Similar results were obtained by Zizy and Awad (2018) in mono-cut Egyptian clover under saline soil.

Table 7: Effect of Potassium fertilizer treatments on fresh forage yield $\left(\mathrm{Kg} / \mathrm{m}^{2}\right.$ for 9 cuts) and total yield (ton/ha) in 2015/2016 season.

\begin{tabular}{|c|c|c|c|c|c|c|c|c|c|c|c|}
\hline \multirow{2}{*}{$\begin{array}{l}\text { Potassium } \\
\text { treatments }\end{array}$} & \multicolumn{2}{|c|}{ Winter } & \multicolumn{2}{|c|}{ Spring } & \multicolumn{3}{|c|}{ Summer } & \multicolumn{2}{|c|}{ Autumn } & \multirow{2}{*}{$\begin{array}{c}\text { Total } \\
\text { yield } \\
\mathrm{Kg} / \mathrm{m}^{2}\end{array}$} & \multirow{2}{*}{$\begin{array}{c}\text { Total } \\
\text { yield } \\
\text { ton/ha }\end{array}$} \\
\hline & $\begin{array}{c}1^{\text {st }} \\
\text { Cut }\end{array}$ & $\begin{array}{l}2^{\text {nd }} \\
\text { Cut }\end{array}$ & $\begin{array}{l}3^{\text {rd }} \\
\text { Cut }\end{array}$ & $\begin{array}{c}4^{\text {th }} \\
\text { Cut }\end{array}$ & $\begin{array}{c}5^{\text {th }} \\
\text { Cut }\end{array}$ & $\begin{array}{c}6^{\text {th }} \\
\text { Cut } \\
\end{array}$ & $\begin{array}{c}7^{\text {th }} \\
\text { Cut }\end{array}$ & $\begin{array}{c}8^{\text {th }} \\
\text { Cut }\end{array}$ & $\begin{array}{c}9^{\text {th }} \\
\text { Cut }\end{array}$ & & \\
\hline $\mathrm{K} 1$ & 0.625 & 0.753 & 1.124 & 1.157 & 1.010 & 0.960 & 0.906 & 0.837 & 0.786 & 8.189 & 81.890 \\
\hline $\mathrm{K} 2$ & 0.740 & 0.858 & 1.359 & 1.568 & 1.429 & 1.189 & 1.230 & 1.207 & 1.115 & 10.697 & 106.970 \\
\hline $\mathrm{K} 3$ & 0.692 & 0.766 & 1.233 & 1.255 & 1.244 & 1.073 & 1.025 & 1.009 & 0.979 & 9.277 & 92.770 \\
\hline $\mathrm{K} 4$ & 0.665 & 0.756 & 1.166 & 1.162 & 1.049 & 0.982 & 0.909 & 0.886 & 0.827 & 8.395 & 83.950 \\
\hline K5 & 0.680 & 0.763 & 1.167 & 1.176 & 1.152 & 0.988 & 0.931 & 0.936 & 0.857 & 8.654 & 86.540 \\
\hline K6 & 0.717 & 0.766 & 1.246 & 1.399 & 1.378 & 1.165 & 1.117 & 1.109 & 1.049 & 9.942 & 99.420 \\
\hline K7 & 0.742 & 0.827 & 1.372 & 1.575 & 1.438 & 1.213 & 1.216 & 1.154 & 1.154 & 10.756 & 107.560 \\
\hline $\operatorname{LSD}_{0.05}$ & 0.030 & 0.053 & 0.143 & 0.121 & 0.119 & 0.085 & 0.080 & 0.090 & 0.065 & & \\
\hline
\end{tabular}


Table 8: Effect of Potassium fertilizer treatments on fresh forage yield (Kg/m $\mathrm{m}^{2}$ for 9 cuts) and total yield (ton/ha) in 2016/2017 season.

\begin{tabular}{|c|c|c|c|c|c|c|c|c|c|c|c|}
\hline \multirow{2}{*}{$\begin{array}{l}\text { Potassium } \\
\text { treatments }\end{array}$} & \multicolumn{2}{|c|}{ Winter } & \multicolumn{2}{|c|}{ Spring } & \multicolumn{2}{|c|}{ Summer } & \multicolumn{3}{|c|}{ Autumn } & \multirow{2}{*}{$\begin{array}{c}\text { Total } \\
\text { yield } \\
\mathbf{K g} / \mathbf{m}^{2} \\
\end{array}$} & \multirow{2}{*}{$\begin{array}{c}\text { Total } \\
\text { yield } \\
\text { ton/ha }\end{array}$} \\
\hline & $1^{\text {st }} \mathrm{Cut}$ & $\begin{array}{l}2^{\text {nd }} \\
\text { Cut }\end{array}$ & $\begin{array}{l}3^{\text {rd }} \\
\text { Cut }\end{array}$ & $\begin{array}{c}4^{\text {th }} \\
\text { Cut } \\
\end{array}$ & $\begin{array}{c}5^{\text {th }} \\
\text { Cut }\end{array}$ & $\begin{array}{c}6^{\text {th }} \\
\text { Cut }\end{array}$ & $\begin{array}{l}7^{\text {th }} \\
\text { Cut } \\
\end{array}$ & $\begin{array}{l}8^{\text {th }} \\
\text { Cut }\end{array}$ & $9^{\text {th }}$ Cut & & \\
\hline $\mathrm{K} 1$ & 0.941 & 1.077 & 1.378 & 1.77 & 1.348 & 1.329 & 1.296 & 1.248 & 1.085 & 11.182 & 111.820 \\
\hline $\mathrm{K} 2$ & 1.090 & 1.251 & 1.791 & 1.871 & 1.705 & 1.716 & 1.678 & 1.587 & 1.450 & 14.145 & 141.450 \\
\hline $\mathrm{K} 3$ & 1.082 & 1.162 & 1.570 & 1.636 & 1.580 & 1.513 & 1.570 & 1.433 & 1.282 & 12.831 & 128.310 \\
\hline $\mathrm{K} 4$ & 1.013 & 1.120 & 1.383 & 1.520 & 1.376 & 1.383 & 1.341 & 1.260 & 1.121 & 11.520 & 115.200 \\
\hline K5 & 1.032 & 1.154 & 1.468 & 1.554 & 1.467 & 1.425 & 1.413 & 1.316 & 1.128 & 11.962 & 119.620 \\
\hline K6 & 1.089 & 1.190 & 1.636 & 1.781 & 1.598 & 1.664 & 1.667 & 1.579 & 1.418 & 13.626 & 136.260 \\
\hline K7 & 1.150 & 1.262 & 1.797 & 1.909 & 1.714 & 1.746 & 1.705 & 1.643 & 1.484 & 14.414 & 144.140 \\
\hline LSD $_{0.05}$ & 0.058 & 0.057 & 0.154 & 0.089 & 0.085 & 0.145 & 0.085 & .091 & 0.135 & & \\
\hline
\end{tabular}

Table 9: Effect of Potassium fertilizer treatments on dry matter (\%) for 9 cuts in 2015/2016 season.

\begin{tabular}{|c|c|c|c|c|c|c|c|c|c|c|}
\hline \multirow{2}{*}{$\begin{array}{l}\text { Potassium } \\
\text { fertilizer } \\
\text { treatments }\end{array}$} & \multicolumn{2}{|c|}{ Winter } & \multicolumn{2}{|c|}{ Spring } & \multicolumn{3}{|c|}{ Summer } & \multicolumn{2}{|c|}{ Autumn } & \multirow{2}{*}{$\begin{array}{l}\text { Means } \\
\text { of Cut }\end{array}$} \\
\hline & $\begin{array}{l}\mathbf{1}^{\text {st }} \\
\text { Cut }\end{array}$ & $\begin{array}{l}2^{\text {nd }} \\
\text { Cut }\end{array}$ & $\begin{array}{l}3^{\text {rd }} \\
\text { Cut }\end{array}$ & $\begin{array}{l}4^{\text {th }} \\
\text { Cut }\end{array}$ & $\begin{array}{c}5^{\text {th }} \\
\text { Cut }\end{array}$ & $\begin{array}{c}\mathbf{6}^{\text {th }} \\
\text { Cut }\end{array}$ & $\begin{array}{l}7^{\text {th }} \\
\text { Cut }\end{array}$ & $\begin{array}{l}8^{\text {th }} \\
\text { Cut }\end{array}$ & $\begin{array}{c}9^{\text {th }} \\
\text { Cut }\end{array}$ & \\
\hline $\mathrm{K} 1$ & 13.95 & 15.91 & 17.91 & 20.15 & 21.58 & 21.66 & 22.97 & 17.37 & 15.79 & 18.587 \\
\hline K2 & 18.29 & 17.96 & 22.84 & 25.13 & 24.30 & 25.60 & 25.28 & 21.46 & 19.14 & 22.222 \\
\hline K3 & 16.93 & 17.19 & 20.16 & 24.15 & 22.80 & 22.69 & 24.23 & 19.76 & 17.33 & 20.582 \\
\hline K4 & 16.16 & 16.32 & 18.50 & 20.74 & 21.66 & 22.51 & 23.35 & 18.61 & 16.01 & 19.317 \\
\hline $\mathrm{K} 5$ & 16.35 & 17.17 & 20.10 & 23.30 & 22.00 & 22.65 & 24.01 & 19.58 & 16.48 & 20.182 \\
\hline K6 & 18.55 & 17.73 & 22.81 & 24.49 & 22.90 & 22.71 & 24.77 & 20.82 & 18.94 & 21.524 \\
\hline K7 & 19.11 & 18.56 & 24.05 & 26.31 & 24.33 & 25.31 & 25.32 & 21.66 & 19.63 & 22.697 \\
\hline LSD $_{0.05}$ & 1.03 & $\mathrm{Ns}$ & 1.07 & 1.11 & 0.99 & 1.06 & $\mathrm{Ns}$ & 1.06 & 1.13 & \\
\hline
\end{tabular}

Table 10: Effect of Potassium fertilizer treatments on dry matter (\%) for 9 cuts in 2016/2017 season.

\begin{tabular}{|c|c|c|c|c|c|c|c|c|c|c|}
\hline \multirow{2}{*}{$\begin{array}{l}\text { Potassium } \\
\text { treatments }\end{array}$} & \multicolumn{2}{|c|}{ Winter } & \multicolumn{2}{|c|}{ Spring } & \multicolumn{3}{|c|}{ Summer } & \multicolumn{2}{|c|}{ Autumn } & \multirow[b]{2}{*}{$\begin{array}{l}\text { Means } \\
\text { of Cut }\end{array}$} \\
\hline & $\begin{array}{l}1^{\text {st }} \\
\text { Cut }\end{array}$ & $\begin{array}{l}2^{\text {nd }} \\
\text { Cut }\end{array}$ & $\begin{array}{l}3^{\text {rd }} \\
\text { Cut }\end{array}$ & $\begin{array}{c}4^{\text {th }} \\
\text { Cut }\end{array}$ & $\begin{array}{c}5^{\text {th }} \\
\text { Cut }\end{array}$ & $\begin{array}{c}\mathbf{6}^{\text {th }} \\
\text { Cut }\end{array}$ & $\begin{array}{l}7^{\text {th }} \\
\text { Cut }\end{array}$ & $\begin{array}{l}8^{\text {th }} \\
\text { Cut }\end{array}$ & $\begin{array}{c}9^{\text {th }} \\
\text { Cut }\end{array}$ & \\
\hline K1 & 14.25 & 16.23 & 18.95 & 20.81 & 22.10 & 22.89 & 21.34 & 21.44 & 19.98 & 19.776 \\
\hline K2 & 18.36 & 18.43 & 25.76 & 26.20 & 24.81 & 25.72 & 25.54 & 25.29 & 22.60 & 23.634 \\
\hline K3 & 17.12 & 18.09 & 21.32 & 24.71 & 23.28 & 23.20 & 24.96 & 24.69 & 21.13 & 22.055 \\
\hline K4 & 16.48 & 16.76 & 19.27 & 21.58 & 22.29 & 23.14 & 23.86 & 22.06 & 20.11 & 20.616 \\
\hline K5 & 16.55 & 17.46 & 19.49 & 23.12 & 22.48 & 23.15 & 24.55 & 23.14 & 20.42 & 21.151 \\
\hline K6 & 18.22 & 18.24 & 22.73 & 25.29 & 23.56 & 24.74 & 25.23 & 25.13 & 22.55 & 22.854 \\
\hline K7 & 19.24 & 18.61 & 25.91 & 26.98 & 24.98 & 25.95 & 25.73 & 26.20 & 22.64 & 24.026 \\
\hline $\operatorname{LSD}_{0.05}$ & 1.01 & Ns & 1.13 & 0.89 & 1.08 & 1.04 & Ns & 0.88 & 1.02 & \\
\hline
\end{tabular}

Table 11: Effect of Potassium fertilizer treatments on dry forage yield $\left(\mathrm{Kg} / \mathrm{m}^{2}\right.$ for 9 cuts) and total yield ton/ha in 2015/2016 season.

\begin{tabular}{|c|c|c|c|c|c|c|c|c|c|c|c|}
\hline \multirow{2}{*}{$\begin{array}{l}\text { Potassium } \\
\text { treatments }\end{array}$} & \multicolumn{2}{|l|}{ Winter } & \multicolumn{2}{|l|}{ Spring } & \multicolumn{3}{|c|}{ Summer } & \multicolumn{2}{|c|}{ Autumn } & \multirow{2}{*}{$\begin{array}{c}\text { Total } \\
\text { yield } \\
\text { Kg/m } / \mathbf{m}^{2}\end{array}$} & \multirow{2}{*}{$\begin{array}{l}\text { Total } \\
\text { yield } \\
\text { ton } / \text { ha }\end{array}$} \\
\hline & $\begin{array}{l}1^{\text {st }} \\
\text { Cut }\end{array}$ & $\begin{array}{l}2^{\text {nd }} \\
\text { Cut }\end{array}$ & $\begin{array}{l}3^{\text {rd }} \\
\text { Cut }\end{array}$ & $\begin{array}{l}4^{\text {th }} \\
\text { Cut }\end{array}$ & $\begin{array}{l}5^{\text {th }} \\
\text { Cut }\end{array}$ & $\begin{array}{l}6^{\text {th }} \\
\text { Cut }\end{array}$ & $\begin{array}{l}7^{\text {th }} \\
\text { Cut }\end{array}$ & $\begin{array}{l}8^{\text {th }} \\
\text { Cut }\end{array}$ & $\begin{array}{l}9^{\text {th }} \\
\text { Cut }\end{array}$ & & \\
\hline K1 & 0.091 & 0.119 & 0.201 & 0.233 & 0.218 & 0.207 & 0.208 & 0.145 & 0.124 & 1.549 & 15.490 \\
\hline $\mathrm{K} 2$ & 0.135 & 0.154 & 0.310 & 0.394 & 0.347 & 0.304 & 0.311 & 0.259 & 0.213 & 2.429 & 24.290 \\
\hline K3 & 0.117 & 0.131 & 0.248 & 0.303 & 0.283 & 0.243 & 0.248 & 0.199 & 0.169 & 1.945 & 19.450 \\
\hline $\mathrm{K} 4$ & 0.107 & 0.123 & 0.215 & 0.241 & 0.227 & 0.218 & 0.212 & 0.161 & 0.132 & 1.639 & 16.390 \\
\hline K5 & 0.111 & 0.131 & 0.234 & 0.274 & 0.253 & 0.223 & 0.223 & 0.183 & 0.141 & 1.776 & 17.760 \\
\hline K6 & 0.131 & 0.135 & 0.284 & 0.342 & 0.315 & 0.264 & 0.276 & 0.230 & 0.198 & 2.181 & 21.810 \\
\hline K7 & 0.141 & 0.153 & 0.330 & 0.414 & 0.350 & 0.307 & 0.307 & 0.263 & 0.226 & 2.494 & 24.940 \\
\hline LSD $_{0.05}$ & 0.005 & 0.004 & 0.020 & 0.026 & 0.025 & 0.045 & 0.025 & 0.009 & 0.013 & & \\
\hline
\end{tabular}


Table 12: Effect of Potassium fertilizer treatments on dry forage yield (Kg/m $\mathrm{m}^{2}$ for 9 cuts) and total yield ton/ha in 2016/2017 season.

\begin{tabular}{|c|c|c|c|c|c|c|c|c|c|c|c|}
\hline \multirow{2}{*}{$\begin{array}{l}\text { Potassium } \\
\text { treatments }\end{array}$} & \multicolumn{2}{|c|}{ Winter } & \multicolumn{2}{|l|}{ Spring } & \multicolumn{3}{|c|}{ Summer } & \multicolumn{2}{|c|}{ Autumn } & \multirow{2}{*}{$\begin{array}{l}\text { Total } \\
\text { yield } \\
\mathrm{Kg} / \mathrm{m}^{2}\end{array}$} & \multirow{2}{*}{$\begin{array}{l}\text { Total } \\
\text { yield } \\
\text { ton/ha }\end{array}$} \\
\hline & $\begin{array}{l}1^{\text {st }} \\
\text { Cut }\end{array}$ & $\begin{array}{l}2^{\text {nd }} \\
\text { Cut }\end{array}$ & $\begin{array}{l}3^{\text {rd }} \\
\text { Cut }\end{array}$ & $\begin{array}{l}4^{\text {th }} \\
\text { Cut }\end{array}$ & $\begin{array}{l}5^{\text {th }} \\
\text { Cut }\end{array}$ & $\begin{array}{l}6^{\text {th }} \\
\text { Cut } \\
\end{array}$ & $\begin{array}{l}7^{\text {th }} \\
\text { Cut }\end{array}$ & $\begin{array}{l}8^{\text {th }} \\
\text { Cut }\end{array}$ & $\begin{array}{l}9^{\text {th }} \\
\text { Cut }\end{array}$ & & \\
\hline $\mathrm{K} 1$ & 0.134 & 0.174 & 0.261 & 0.307 & 0.297 & 0.304 & 0.276 & 0.267 & 0.216 & 2.240 & 22.400 \\
\hline $\mathrm{K} 2$ & 0.200 & 0.230 & 0.461 & 0.490 & 0.423 & 0.441 & 0.428 & 0.401 & 0.327 & 3.405 & 34.050 \\
\hline K3 & 0.185 & 0.210 & 0.334 & 0.404 & 0.367 & 0.352 & 0.391 & 0.353 & 0.270 & 2.871 & 28.710 \\
\hline K4 & 0.167 & 0.187 & 0.266 & 0.328 & 0.306 & 0.320 & 0.320 & 0.277 & 0.225 & 2.399 & 23.99 \\
\hline K5 & & & & & & & & & & & 25.610 \\
\hline K6 & 0.198 & 0.217 & 0.371 & 0.450 & 0.376 & 0.411 & 0.420 & 0.396 & 0.319 & 3.163 & 31.630 \\
\hline K7 & 0.221 & 0.234 & 0.465 & 0.515 & 0.428 & 0.453 & 0.438 & 0.430 & 0.336 & 3.523 & 35.230 \\
\hline LSD $_{0.05}$ & 0.016 & $\mathrm{Ns}$ & 0.077 & 0.039 & 0.034 & 0.042 & 0.027 & 0.033 & 0.030 & & \\
\hline
\end{tabular}

\section{Chemical and physiological traits:}

Data of relative water content (RWC \%), $\mathrm{Na}^{+}$, $\mathrm{K}^{+}, \mathrm{K}^{+} / \mathrm{Na}^{+}$ratio as well as protein content are presented in Tables $(13,14,15,16,17,18,19,20$, 21 and 22). Relative water content was determined to give an indication on the plant water dehydration status during exposure to salinity which reflects the balance between water supply to the leaf and transpiration rate. Results in Tables (13 and 14) showed that there were significant increases for RWC\% with increasing potassium fertilization. The highest values were obtained from plants received $28.6 \mathrm{KgK}_{2} \mathrm{O} / \mathrm{ha}+2 \% \mathrm{~K}_{2} \mathrm{O}, 57.14 \mathrm{KgK}_{2} \mathrm{O} /$ ha as well as $28.6 \mathrm{KgK}_{2} \mathrm{O} / \mathrm{ha}$ with insignificant difference in all cuttings in the first and second year, except the $1^{\text {st }}$ and $2^{\text {nd }}$ cuts in the first year and in the $1^{\text {st }}, 5^{\text {th }}$ and $9^{\text {th }}$ cuts in the second year in which plants received $28.6 \mathrm{KgK}_{2} \mathrm{O} / \mathrm{ha}+2 \% \mathrm{~K}_{2} \mathrm{O}$ and $57.14 \mathrm{KgK}_{2} \mathrm{O} /$ ha gave the highest RWC values with insignificant difference while plants received $28.6 \mathrm{KgK}_{2} \mathrm{O} / \mathrm{ha}$ $+2 \% \mathrm{~K}_{2} \mathrm{O}$ and $28.6 \mathrm{KgK}_{2} \mathrm{O} / \mathrm{ha}+1 \% \mathrm{~K}_{2} \mathrm{O}$ gave the highest RWC values in $5^{\text {th }}$ cut in second year. Such results may be due to the fact that under salt stress condition and water deficit, $\mathrm{K}^{+}$is pumped out from the guard cell, allowing the pores to close and controlling the evapotranspiration of water and protects the plants. In addition, the osmotic gradient produced due to the accumulation of $\mathrm{K}^{+}$in the roots helps to draw water into the root cells (Hasanuzzaman et al., 2018). These results are in harmony with those obtained by Abdo Fatma and Anton (2009) in sesame plants, Heba and Mary (2017) in barely plants and El-sharkawy et al (2017) in alfalfa.

Data of potassium, sodium and $\mathrm{K}^{+} / \mathrm{Na}^{+}$ratio in Tables (15, 16, 17, 18, 19 and 20) showed that potassium fertilization recorded significant effects concerning those traits. The highest $\mathrm{K}^{+}$values were achieved when plants received $28.6 \mathrm{KgK}_{2} \mathrm{O} / \mathrm{ha}+$ $2 \% \mathrm{~K}_{2} \mathrm{O}, \quad 57.14 \mathrm{KgK}_{2} \mathrm{O} / \mathrm{ha}$ and $28.6 \mathrm{KgK}_{2} \mathrm{O} / \mathrm{ha}+$ $1 \% \mathrm{~K}_{2} \mathrm{O}$ with insignificant difference in all cuts in both years except the $1^{\text {st }}, 2^{\text {nd }}$ cuts in the first year and the $4^{\text {th }}$ cut in both years which recorded the highest $\mathrm{K}^{+}$value from plants received $28.6 \mathrm{KgK}_{2} \mathrm{O} / \mathrm{ha}+2 \% \mathrm{~K}_{2} \mathrm{O}$ and $57.14 \mathrm{Kg} \mathrm{K}_{2} \mathrm{O} / \mathrm{ha}$ with in significant difference.

Concerning $\mathrm{K}^{+} / \mathrm{Na}^{+}$ratio, the highest values were obtained from plants received $28.6 \mathrm{KgK}_{2} \mathrm{O}+$ $2 \% \mathrm{~K}_{2} \mathrm{O}, \quad 57.14 \mathrm{KgK}_{2} \mathrm{O} / \mathrm{ha}$ and $28.6 \mathrm{KgK}_{2} \mathrm{O} / \mathrm{ha}+$ $1 \% \mathrm{~K}_{2} \mathrm{O}$ with insignificant difference in all cuts in both years except $4^{\text {th }}$ and $6^{\text {th }}$ in the first year and $1^{\text {st }}$ cuts for both years which gave the highest values from plants received $28.6 \mathrm{KgK}_{2} \mathrm{O} / \mathrm{ha}+2 \% \mathrm{~K}_{2} \mathrm{O}$ and $57.14 \mathrm{~K}_{2} \mathrm{O} / \mathrm{ha}$ with insignificant difference. On the other hand, the reverse trend was obtained with respect to $\mathrm{Na}^{+}$concentration which significantly decreased by increasing $\mathrm{K}^{+}$fertilization. The highest values of $\mathrm{Na}^{+}$concentration were obtained when plants were untreated by potassium fertilization in all cuts in both years while the lower values were obtained from plants received $28.6 \mathrm{KgK}_{2} \mathrm{O}+2 \% \mathrm{~K}_{2} \mathrm{O}$, $57.14 \mathrm{KgK}_{2} \mathrm{O} / \mathrm{ha}$ as well as $28.6 \mathrm{KgK}_{2} \mathrm{O}+1 \% \mathrm{~K}_{2} \mathrm{O}$. Under salinity stress, the osmotic effect and ion toxicity decreases nutrient uptake and translocation especially that of $\mathrm{K}$, the sodium ion competes with $\mathrm{K}^{+}$for major binding sites during key metabolic processes; this competition disturbs the plant metabolism. Also, salinity induces membrane depolarization and decreases the membrane integrity, which results in $\mathrm{K}^{+}$leakage through depolarization-activated outward-rectifying (KOR) $\mathrm{K}$ channels (Shabala and Cuin 2008), thus higher applications of potassium increase the $\mathrm{K}^{+}$content in plant cells and reduce the $\mathrm{Na}^{+}$concentration, which increase $\mathrm{K}^{+} / \mathrm{Na}^{+}$ratio which have vital roles in plant $\mathrm{Na}^{+}$tolerance. Similar results were obtained by Khorshidi et al (2009) in alfalfa and Heba and Mary (2017) in barely.

With regard to protein content, data in Tables (21 and 22) showed that potassium fertilization significantly affected protein content in all cuts in both years except the $1^{\text {st }}, 4^{\text {th }}$ and $5^{\text {th }}$ cuts in the first year. 
Table 13: Effect of Potassium fertilizer treatments on relative water content (RWC \%), for 9 cuts in 2015/2016 season.

\begin{tabular}{|c|c|c|c|c|c|c|c|c|c|c|}
\hline \multirow{2}{*}{$\begin{array}{l}\text { Potassium } \\
\text { treatments }\end{array}$} & \multicolumn{2}{|c|}{ Winter } & \multicolumn{2}{|c|}{ Spring } & \multicolumn{3}{|c|}{ Summer } & \multicolumn{2}{|c|}{ Autumn } & \multirow[b]{2}{*}{$\begin{array}{l}\text { Means } \\
\text { of Cut }\end{array}$} \\
\hline & $\begin{array}{c}1^{\text {st }} \\
\text { Cut }\end{array}$ & $\begin{array}{c}2^{\text {nd }} \\
\text { Cut }\end{array}$ & $\begin{array}{c}3^{\text {rd }} \\
\text { Cut }\end{array}$ & $\begin{array}{c}4^{\text {th }} \\
\text { Cut }\end{array}$ & $\begin{array}{c}5^{\text {th }} \\
\text { Cut }\end{array}$ & $\begin{array}{c}\mathbf{6}^{\text {th }} \\
\text { Cut }\end{array}$ & $\begin{array}{c}7^{\text {th }} \\
\text { Cut }\end{array}$ & $\begin{array}{c}8^{\text {th }} \\
\text { Cut }\end{array}$ & $\begin{array}{c}9^{\text {th }} \\
\text { Cut }\end{array}$ & \\
\hline K1 & 70.49 & 73.56 & 76.31 & 75.06 & 70.30 & 68.50 & 68.85 & 72.60 & 72.34 & 72.00 \\
\hline $\mathrm{K} 2$ & 79.13 & 80.41 & 86.87 & 85.20 & 82.33 & 79.13 & 78.97 & 79.99 & 81.40 & 81.49 \\
\hline K3 & 75.80 & 77.62 & 84.35 & 80.91 & 78.15 & 74.20 & 74.66 & 77.15 & 79.15 & 77.99 \\
\hline K4 & 70.88 & 75.41 & 79.67 & 77.15 & 72.61 & 70.13 & 71.06 & 74.11 & 77.12 & 74.23 \\
\hline K5 & 73.12 & 75.94 & 81.90 & 78.62 & 75.40 & 73.22 & 74.22 & 76.03 & 78.66 & 76.34 \\
\hline K6 & 77.96 & 79.02 & 85.94 & 85.04 & 81.19 & 78.98 & 79.04 & 80.07 & 81.96 & 81.02 \\
\hline K7 & 78.91 & 81.18 & 87.99 & 86.13 & 83.40 & 79.64 & 79.88 & 80.22 & 82.44 & 82.19 \\
\hline $\operatorname{LSD}_{0.05}$ & 1.15 & 1.24 & 1.20 & 1.41 & 1.33 & 1.26 & 1.12 & 1.09 & 1.10 & \\
\hline
\end{tabular}

Table 14: Effect of Potassium fertilizer treatments on relative water content (RWC \%), for 9 cuts in 2016/2017 season.

\begin{tabular}{|c|c|c|c|c|c|c|c|c|c|c|}
\hline \multirow{2}{*}{$\begin{array}{l}\text { Potassium } \\
\text { treatments }\end{array}$} & \multicolumn{2}{|c|}{ Winter } & \multicolumn{2}{|c|}{ Spring } & \multicolumn{3}{|c|}{ Summer } & \multicolumn{2}{|c|}{ Autumn } & \multirow{2}{*}{$\begin{array}{l}\text { Means } \\
\text { of Cut }\end{array}$} \\
\hline & $\begin{array}{c}\mathbf{1}^{\text {st }} \\
\text { Cut }\end{array}$ & $\begin{array}{c}2^{\text {nd }} \\
\text { Cut }\end{array}$ & $\begin{array}{c}3^{\text {rd }} \\
\text { Cut }\end{array}$ & $\begin{array}{c}4^{\text {th }} \\
\text { Cut }\end{array}$ & $\begin{array}{c}5^{\text {th }} \\
\text { Cut }\end{array}$ & $\begin{array}{c}\mathbf{6}^{\text {th }} \\
\text { Cut }\end{array}$ & $\begin{array}{c}7^{\text {th }} \\
\text { Cut }\end{array}$ & $\begin{array}{c}8^{\text {th }} \\
\text { Cut }\end{array}$ & $\begin{array}{c}9^{\text {th }} \\
\text { Cut }\end{array}$ & \\
\hline K1 & 74.11 & 74.93 & 75.90 & 73.81 & 70.11 & 66.11 & 67.54 & 71.44 & 73.60 & 71.95 \\
\hline $\mathrm{K} 2$ & 81.96 & 82.30 & 84.88 & 81.66 & 78.40 & 79.20 & 79.66 & 80.66 & 81.23 & 81.10 \\
\hline K3 & 78.71 & 78.97 & 79.66 & 78.20 & 73.88 & 75.15 & 71.20 & 78.44 & 78.11 & 76.92 \\
\hline K4 & 74.96 & 77.13 & 77.10 & 76.20 & 72.08 & 69.20 & 68.60 & 72.16 & 75.81 & 73.69 \\
\hline K5 & 77.51 & 78.93 & 79.23 & 77.31 & 74.20 & 74.44 & 72.14 & 77.69 & 77.69 & 76.57 \\
\hline K6 & 80.44 & 82.04 & 84.90 & 82.30 & 79.66 & 79.86 & 80.14 & 80.98 & 82.40 & 81.41 \\
\hline K7 & 82.40 & 83.33 & 85.23 & 82.48 & 80.13 & 80.61 & 81.08 & 81.40 & 82.66 & 82.14 \\
\hline $\operatorname{LSD}_{0.05}$ & 1.20 & 1.14 & 1.06 & 1.10 & 1.09 & 1.11 & 1.05 & 1.00 & 1.12 & \\
\hline
\end{tabular}

Table 15: Effect of Potassium fertilizer treatments on potassium (\%), for 9 cuts in 2015/2016 season.

\begin{tabular}{|c|c|c|c|c|c|c|c|c|c|c|}
\hline \multirow{2}{*}{$\begin{array}{l}\text { Potassium } \\
\text { treatments }\end{array}$} & \multicolumn{2}{|c|}{ Winter } & \multicolumn{2}{|c|}{ Spring } & \multicolumn{3}{|c|}{ Summer } & \multicolumn{2}{|c|}{ Autumn } & \multirow[b]{2}{*}{$\begin{array}{l}\text { Means } \\
\text { of Cut }\end{array}$} \\
\hline & $\begin{array}{c}\mathbf{1}^{\text {st }} \\
\text { Cut }\end{array}$ & $\begin{array}{l}2^{\text {nd }} \\
\text { Cut }\end{array}$ & $\begin{array}{c}3^{\text {rd }} \\
\text { Cut }\end{array}$ & $\begin{array}{l}4^{\text {th }} \\
\text { Cut }\end{array}$ & $\begin{array}{c}5^{\text {th }} \\
\text { Cut }\end{array}$ & $\begin{array}{c}\mathbf{6}^{\text {th }} \\
\text { Cut }\end{array}$ & $\begin{array}{c}7^{\text {th }} \\
\text { Cut }\end{array}$ & $\begin{array}{c}8^{\text {th }} \\
\text { Cut }\end{array}$ & $\begin{array}{c}9^{\text {th }} \\
\text { Cut }\end{array}$ & \\
\hline K1 & 1.563 & 1.580 & 1.634 & 1.641 & 1.436 & 1.355 & 1.39 & 1.572 & 1.586 & 1.528 \\
\hline $\mathrm{K} 2$ & 2.587 & 2.514 & 2.361 & 2.459 & 2.540 & 2.550 & 2.557 & 2.515 & 2.530 & 2.512 \\
\hline K3 & 2.330 & 2.466 & 2.110 & 2.330 & 2.421 & 2.472 & 2.436 & 2.381 & 2.413 & 2.373 \\
\hline K4 & 2.064 & 2.149 & 1.950 & 1.989 & 2.248 & 1.939 & 2.177 & 2.205 & 2.209 & 2.103 \\
\hline K5 & 2.101 & 2.247 & 1.979 & 2.008 & 2.392 & 2.217 & 2.355 & 2.224 & 2.353 & 2.208 \\
\hline K6 & 2.401 & 2.501 & 2.352 & 2.338 & 2.541 & 2.552 & 2.562 & 2.573 & 2.555 & 2.486 \\
\hline K7 & 2.590 & 2.593 & 2.369 & 2.469 & 2.556 & 2.567 & 2.569 & 2.576 & 2.594 & 2.542 \\
\hline $\operatorname{LSD}_{0.05}$ & 0.093 & 0.86 & 0.090 & 0.098 & 0.077 & 0.098 & 0.063 & 0.060 & 0.057 & \\
\hline
\end{tabular}

Table 16: Effect of Potassium fertilizer treatments on potassium (\%), for 9 cuts in 2016/2017 season.

\begin{tabular}{|c|c|c|c|c|c|c|c|c|c|c|}
\hline \multirow{2}{*}{$\begin{array}{l}\text { Potassiu } \\
\text { m } \\
\text { treatme } \\
\text { nts }\end{array}$} & \multicolumn{2}{|l|}{ Winter } & \multicolumn{2}{|l|}{ Spring } & \multicolumn{3}{|c|}{ Summer } & \multicolumn{2}{|c|}{ Autumn } & \multirow[b]{2}{*}{$\begin{array}{l}\text { Means } \\
\text { of Cut }\end{array}$} \\
\hline & $\mathbf{1}^{\text {st }}$ Cut & $\begin{array}{l}2^{\text {nd }} \\
\text { Cut }\end{array}$ & $\begin{array}{l}3^{\text {rd }} \\
\text { Cut }\end{array}$ & $\begin{array}{l}4^{\text {th }} \\
\text { Cut }\end{array}$ & $\begin{array}{l}5^{\text {th }} \\
\text { Cut }\end{array}$ & $\begin{array}{l}\text { 6 }^{\text {th }} \\
\text { Cut }\end{array}$ & $\begin{array}{l}7^{\text {th }} \\
\text { Cut }\end{array}$ & $\begin{array}{l}8^{\text {th }} \\
\text { Cut }\end{array}$ & $\begin{array}{l}9^{\text {th }} \\
\text { Cut }\end{array}$ & \\
\hline $\mathrm{K} 1$ & 1.664 & 1.673 & 1.721 & 1.654 & 1.552 & 1.392 & 1.246 & 1.496 & 1.561 & 1.551 \\
\hline $\mathrm{K} 2$ & 2.532 & 2.671 & 2.620 & 2.742 & 2.591 & 2.694 & 2.547 & 2.576 & 2.726 & 2.633 \\
\hline K3 & 2.451 & 2.590 & 2.337 & 2.430 & 2.338 & 2.450 & 2.438 & 2.411 & 2.587 & 2.448 \\
\hline K4 & 2.135 & 2.236 & 2.109 & 2.117 & 2.217 & 2.387 & 2.173 & 2.215 & 2.246 & 2.203 \\
\hline K5 & 2.240 & 2.358 & 2.151 & 2.154 & 2.306 & 2.419 & 2.329 & 2.375 & 2.388 & 2.302 \\
\hline K6 & 2.553 & 2.603 & 2.582 & 2.625 & 2.604 & 2.653 & 2.511 & 2.532 & 2.715 & 2.597 \\
\hline K7 & 2.579 & 2.694 & 2.665 & 2.751 & 2.690 & 2.711 & 2.682 & 2.613 & 2.731 & 2.679 \\
\hline LSD $_{0.05}$ & 0.080 & 0.069 & 0.049 & 0.061 & 0.052 & 0.093 & 0.070 & 0.089 & 0.071 & \\
\hline
\end{tabular}


Table 17: Effect of Potassium fertilizer treatments on sodium (\%), for 9 cuts in 2015/2016 season.

\begin{tabular}{|c|c|c|c|c|c|c|c|c|c|c|}
\hline \multirow{2}{*}{$\begin{array}{l}\text { Potassium } \\
\text { treatments }\end{array}$} & \multicolumn{2}{|c|}{ Winter } & \multicolumn{2}{|c|}{ Spring } & \multicolumn{3}{|c|}{ Summer } & \multicolumn{2}{|c|}{ Autumn } & \multirow[b]{2}{*}{$\begin{array}{c}\text { Means of } \\
\text { Cut }\end{array}$} \\
\hline & $\begin{array}{c}1^{\text {st }} \\
\text { Cut }\end{array}$ & $\begin{array}{l}\mathbf{2}^{\text {nd }} \\
\text { Cut }\end{array}$ & $\begin{array}{l}3^{\text {rd }} \\
\text { Cut }\end{array}$ & $\begin{array}{c}4^{\text {th }} \\
\text { Cut }\end{array}$ & $\begin{array}{l}5^{\text {th }} \\
\text { Cut }\end{array}$ & $\begin{array}{l}6^{\text {th }} \\
\text { Cut }\end{array}$ & $\begin{array}{l}7^{\text {th }} \\
\text { Cut }\end{array}$ & $\begin{array}{l}\mathbf{8}^{\text {th }} \\
\text { Cut }\end{array}$ & $\begin{array}{l}9^{\text {th }} \\
\text { Cut }\end{array}$ & \\
\hline K1 & 0.261 & 0.257 & 0.260 & 0.259 & 0.268 & 0.258 & 0.266 & 0.271 & 0.270 & 0.263 \\
\hline K2 & 0.203 & 0.212 & 0.211 & 0.203 & 0.218 & 0.219 & 0.221 & 0.213 & 0.220 & 0.213 \\
\hline K3 & 0.221 & 0.237 & 0.219 & 0.235 & 0.240 & 0.226 & 0.243 & 0.240 & 0.253 & 0.234 \\
\hline K4 & 0.256 & 0.243 & 0.237 & 0.255 & 0.244 & 0.231 & 0.250 & 0.251 & 0.258 & 0.247 \\
\hline K5 & 0.237 & 0.241 & 0.231 & 0.241 & 0.241 & 0.229 & 0.245 & 0.242 & 0.255 & 0.240 \\
\hline K6 & 0.208 & 0.215 & 0.214 & 0.205 & 0.221 & 0.214 & 0.225 & 0.225 & 0.221 & 0.216 \\
\hline K7 & 0.209 & 0.211 & 0.209 & 0.201 & 0.217 & 0.203 & 0.220 & 0.218 & 0.216 & 0.212 \\
\hline $\operatorname{LSD}_{0.05}$ & 0.017 & 0.011 & 0.021 & 0.012 & 0.019 & 0.017 & 0.013 & 0.016 & 0.011 & \\
\hline
\end{tabular}

Table 18: Effect of Potassium fertilizer treatments on sodium (\%), for 9 cuts in 2016/2017 season.

\begin{tabular}{|c|c|c|c|c|c|c|c|c|c|c|}
\hline \multirow{2}{*}{$\begin{array}{l}\text { Potassium } \\
\text { treatments }\end{array}$} & \multicolumn{2}{|c|}{ Winter } & \multicolumn{2}{|c|}{ Spring } & \multicolumn{3}{|c|}{ Summer } & \multicolumn{2}{|c|}{ Autumn } & \multirow[b]{2}{*}{$\begin{array}{l}\text { Means of } \\
\text { Cut }\end{array}$} \\
\hline & $\begin{array}{l}1^{\text {st }} \\
\text { Cut }\end{array}$ & $\begin{array}{l}2^{\text {nd }} \\
\text { Cut }\end{array}$ & $\begin{array}{l}3^{\text {rd }} \\
\text { Cut }\end{array}$ & $\begin{array}{c}4^{\text {th }} \\
\text { Cut }\end{array}$ & $\begin{array}{l}5^{\text {th }} \\
\text { Cut }\end{array}$ & $\begin{array}{c}\mathbf{6}^{\text {th }} \\
\text { Cut }\end{array}$ & $\begin{array}{l}7^{\text {th }} \\
\text { Cut }\end{array}$ & $\begin{array}{l}8^{\text {th }} \\
\text { Cut }\end{array}$ & $\begin{array}{c}9^{\text {th }} \\
\text { Cut }\end{array}$ & \\
\hline K1 & 0.246 & 0.274 & 0.271 & 0.276 & 0.282 & 0.291 & 0.293 & 0.266 & 0.259 & 0.273 \\
\hline K2 & 0.211 & 0.220 & 0.223 & 0.234 & 0.240 & 0.249 & 0.253 & 0.213 & 0.212 & 0.228 \\
\hline K3 & 0.216 & 0.243 & 0.249 & 0.250 & 0.251 & 0.256 & $0 . .262$ & 0.242 & 0.231 & 0.242 \\
\hline K4 & 0.225 & 0.251 & 0.255 & 0.259 & 0.264 & 0.266 & 0.267 & 0.249 & 0.243 & 0.253 \\
\hline K5 & 0.220 & 0.250 & 0.251 & 0.254 & 0.261 & 0.260 & 0.263 & 0.245 & 0.240 & 0.249 \\
\hline K6 & 0.211 & 0.223 & 0.227 & 0.238 & 0.238 & 0.247 & 0.251 & 0.219 & 0.220 & 0.230 \\
\hline K7 & 0.209 & 0.208 & 0.211 & 0.233 & 0.236 & 0.245 & 0.246 & 0.214 & 0.211 & 0.223 \\
\hline $\operatorname{LSD}_{0.05}$ & 0.019 & 0.20 & 0.015 & 0.014 & 0.017 & 0.020 & 0.019 & 0.015 & 0.013 & \\
\hline
\end{tabular}

Table 19: Effect of Potassium fertilizer treatments on potassium/ sodium ratio, for 9 cuts in 2015/2016 season.

\begin{tabular}{|c|c|c|c|c|c|c|c|c|c|c|}
\hline \multirow{2}{*}{$\begin{array}{l}\text { Potassium } \\
\text { treatments }\end{array}$} & \multicolumn{2}{|c|}{ Winter } & \multicolumn{2}{|c|}{ Spring } & \multicolumn{3}{|c|}{ Summer } & \multicolumn{2}{|c|}{ Autumn } & \multirow{2}{*}{$\begin{array}{l}\text { Means } \\
\text { of Cut }\end{array}$} \\
\hline & $1^{\text {st }} \mathrm{Cut}$ & $\begin{array}{l}2^{\text {nd }} \\
\text { Cut }\end{array}$ & $\begin{array}{c}3^{\text {rd }} \\
\text { Cut }\end{array}$ & $\begin{array}{c}4^{\text {th }} \\
\text { Cut }\end{array}$ & $\begin{array}{c}5^{\text {th }} \\
\text { Cut }\end{array}$ & $\begin{array}{c}6^{\text {th }} \\
\text { Cut }\end{array}$ & $\begin{array}{c}7^{\text {th }} \\
\text { Cut }\end{array}$ & $\begin{array}{c}8^{\text {th }} \\
\text { Cut }\end{array}$ & $\begin{array}{c}9^{\text {th }} \\
\text { Cut }\end{array}$ & \\
\hline $\mathrm{K} 1$ & 5.988 & 6.147 & 6.284 & 6.335 & 5.358 & 5.251 & 5.225 & 5.800 & 5.874 & 5.806 \\
\hline $\mathrm{K} 2$ & 12.709 & 11.858 & 11.189 & 12.113 & 11.651 & 11.643 & 11.570 & 11.807 & 11.500 & 11.782 \\
\hline K3 & 10.543 & 10.405 & 9.634 & 9.914 & 10.087 & 10.938 & 10.024 & 9.920 & 9.537 & 10.111 \\
\hline K4 & 8.062 & 8.843 & 8.227 & 7.800 & 9.213 & 8.393 & 8.708 & 8.784 & 8.562 & 8.510 \\
\hline K5 & 8.865 & 9.323 & 8.567 & 8.331 & 9.925 & 9.681 & 9.612 & 9.190 & 9.227 & 9.191 \\
\hline K6 & 11.543 & 11.632 & 10.990 & 11.404 & 11.497 & 11.925 & 11.386 & 11.435 & 11.561 & 11.485 \\
\hline K7 & 12.392 & 11.990 & 11.334 & 12.283 & 11.778 & 12.645 & 11.677 & 11.816 & 12.009 & 11.991 \\
\hline LSD $_{0.05}$ & 0.820 & 0.770 & 0.833 & 0.961 & 0.710 & 1.004 & 0.993 & 1.000 & 0.862 & \\
\hline
\end{tabular}

Table 20: Effect of Potassium fertilizer treatments on potassium/ sodium ratio, for 9 cuts in 2016/2017 season.

\begin{tabular}{|c|c|c|c|c|c|c|c|c|c|c|}
\hline \multirow{2}{*}{$\begin{array}{l}\text { Potassium } \\
\text { treatments }\end{array}$} & \multicolumn{2}{|c|}{ Winter } & \multicolumn{2}{|c|}{ Spring } & \multicolumn{3}{|c|}{ Summer } & \multicolumn{2}{|c|}{ Autumn } & \multirow[b]{2}{*}{$\begin{array}{l}\text { Means } \\
\text { of Cut }\end{array}$} \\
\hline & $\begin{array}{c}1^{\text {st }} \\
\text { Cut }\end{array}$ & $\begin{array}{c}2^{\text {nd }} \\
\text { Cut }\end{array}$ & $\begin{array}{l}3^{\text {rd }} \\
\text { Cut }\end{array}$ & $\begin{array}{c}4^{\text {th }} \\
\text { Cut }\end{array}$ & $\begin{array}{c}5^{\text {th }} \\
\text { Cut }\end{array}$ & $\begin{array}{c}\text { 6 }^{\text {th }} \\
\text { Cut }\end{array}$ & $\begin{array}{c}7^{\text {th }} \\
\text { Cut }\end{array}$ & $\begin{array}{c}8^{\text {th }} \\
\text { Cut }\end{array}$ & $\begin{array}{c}9^{\text {th }} \\
\text { Cut }\end{array}$ & \\
\hline $\mathrm{K} 1$ & 6.764 & 6.105 & 6.350 & 5.992 & 5.503 & 4.783 & 4.313 & 5.624 & 6.027 & 5.717 \\
\hline $\mathrm{K} 2$ & 12.000 & 12.140 & 11.748 & 11.717 & 10.795 & 10.819 & 10.067 & 12.093 & 12.585 & 11.551 \\
\hline $\mathrm{K} 3$ & 11.347 & 10.658 & 9.385 & 9.720 & 9.314 & 9.570 & 9.305 & 9.962 & 11.199 & 10.051 \\
\hline K4 & 9.488 & 8.908 & 8.270 & 8.173 & 8.397 & 8.973 & 8.138 & 8.895 & 9.242 & 8.720 \\
\hline $\mathrm{K} 5$ & 10.181 & 9.436 & 8.569 & 8.840 & 8.835 & 9.303 & 8.855 & 9.620 & 9.950 & 9.287 \\
\hline K6 & 12.099 & 11.672 & 11.374 & 11.029 & 10.941 & 10.740 & 10.003 & 11.561 & 12.340 & 11.306 \\
\hline K7 & 12.339 & 12.952 & 12.630 & 11.806 & 11.398 & 11.065 & 10.902 & 12.210 & 12.943 & 12.027 \\
\hline $\operatorname{LSD}_{0.05}$ & 0.650 & 0.833 & 0.890 & 0.712 & 0.904 & 0.710 & 0.695 & 0.811 & 0.900 & \\
\hline
\end{tabular}


Table 21: Effect of Potassium fertilizer treatments on crude protein (\%), for 9 cuts in 2015/2016 season.

\begin{tabular}{|c|c|c|c|c|c|c|c|c|c|c|}
\hline \multirow{2}{*}{$\begin{array}{l}\text { Potassium } \\
\text { treatments }\end{array}$} & \multicolumn{2}{|c|}{ Winter } & \multicolumn{2}{|c|}{ Spring } & \multicolumn{3}{|c|}{ Summer } & \multicolumn{2}{|c|}{ Autumn } & \multirow{2}{*}{$\begin{array}{l}\text { Means } \\
\text { of Cut }\end{array}$} \\
\hline & $\begin{array}{l}1^{\text {st }} \\
\text { Cut }\end{array}$ & $\begin{array}{l}2^{\text {nd }} \\
\text { Cut }\end{array}$ & $\begin{array}{l}3^{\text {rd }} \\
\text { Cut }\end{array}$ & $\begin{array}{c}4^{\text {th }} \\
\text { Cut }\end{array}$ & $\begin{array}{c}5^{\text {th }} \\
\text { Cut }\end{array}$ & $\begin{array}{c}\mathbf{6}^{\text {th }} \\
\text { Cut }\end{array}$ & $\begin{array}{c}7^{\text {th }} \\
\text { Cut }\end{array}$ & $\begin{array}{c}8^{\text {th }} \\
\text { Cut }\end{array}$ & $\begin{array}{c}9^{\text {th }} \\
\text { Cut }\end{array}$ & \\
\hline K1 & 19.04 & 19.87 & 20.07 & 20.14 & 15.19 & 15.21 & 15.17 & 16.32 & 17.24 & 17.58 \\
\hline $\mathrm{K} 2$ & 22.63 & 23.73 & 24.18 & 22.60 & 17.93 & 17.78 & 17.93 & 20.68 & 21.18 & 20.96 \\
\hline K3 & 20.88 & 22.42 & 22.33 & 21.78 & 16.56 & 16.85 & 16.74 & 19.27 & 19.84 & 19.63 \\
\hline K4 & 20.05 & 21.55 & 21.39 & 21.02 & 16.02 & 16.07 & 16.01 & 18.16 & 18.08 & 18.70 \\
\hline K5 & 20.27 & 22.04 & 21.91 & 21.64 & 16.96 & 16.58 & 16.48 & 18.64 & 18.57 & 19.23 \\
\hline K6 & 21.34 & 23.13 & 23.01 & 22.12 & 17.05 & 17.26 & 17.16 & 20.09 & 20.73 & 20.21 \\
\hline K7 & 22.19 & 24.81 & 24.75 & 23.33 & 18.57 & 19.58 & 19.54 & 20.87 & 21.61 & 21.69 \\
\hline $\operatorname{LSD}_{0.05}$ & Ns & 1.01 & 1.13 & Ns & Ns & 0.96 & 1.63 & 0.80 & 0.88 & \\
\hline
\end{tabular}

Table 22: Effect of Potassium fertilizer treatments on crude protein (\%), for 9 cuts in 2016/2017 season.

\begin{tabular}{|c|c|c|c|c|c|c|c|c|c|c|}
\hline \multirow{2}{*}{$\begin{array}{l}\text { Potassium } \\
\text { treatments }\end{array}$} & \multicolumn{2}{|c|}{ Winter } & \multicolumn{2}{|c|}{ Spring } & \multicolumn{3}{|c|}{ Summer } & \multicolumn{2}{|c|}{ Autumn } & \multirow{2}{*}{$\begin{array}{l}\text { Means } \\
\text { of Cut }\end{array}$} \\
\hline & $\begin{array}{l}1^{\text {st }} \\
\text { Cut }\end{array}$ & $\begin{array}{l}2^{\text {nd }} \\
\text { Cut }\end{array}$ & $\begin{array}{c}\mathbf{3}^{\text {rd }} \\
\text { Cut }\end{array}$ & $\begin{array}{c}4^{\text {th }} \\
\text { Cut }\end{array}$ & $\begin{array}{c}5^{\text {th }} \\
\text { Cut }\end{array}$ & $\begin{array}{c}\mathbf{6}^{\text {th }} \\
\text { Cut }\end{array}$ & $\begin{array}{c}7^{\text {th }} \\
\text { Cut }\end{array}$ & $\begin{array}{c}8^{\text {th }} \\
\text { Cut }\end{array}$ & $\begin{array}{c}9^{\text {th }} \\
\text { Cut }\end{array}$ & \\
\hline K1 & 20.09 & 21.11 & 21.25 & 21.37 & 14.06 & 14.31 & 13.54 & 17.29 & 17.34 & 17.81 \\
\hline $\mathrm{K} 2$ & 24.37 & 24.04 & 25.67 & 25.72 & 17.81 & 17.58 & 15.76 & 20.87 & 22.26 & 21.56 \\
\hline K3 & 22.77 & 22.86 & 23.21 & 23.44 & 16.47 & 16.46 & 15.37 & 18.72 & 19.28 & 19.84 \\
\hline K4 & 21.31 & 22.03 & 22.08 & 22.21 & 15.11 & 15.14 & 14.21 & 18.08 & 18.04 & 18.69 \\
\hline K5 & 22.19 & 22.59 & 22.46 & 22.57 & 15.66 & 15.72 & 14.72 & 18.54 & 18.67 & 19.23 \\
\hline K6 & 23.55 & 23.13 & 24.06 & 24.08 & 17.04 & 17.03 & 15.85 & 19.46 & 20.31 & 20.50 \\
\hline K7 & 24.81 & 25.68 & 26.33 & 26.15 & 19.78 & 18.92 & 17.68 & 21.71 & 22.59 & 22.62 \\
\hline $\mathrm{LSD}_{0.05}$ & 0.81 & 0.99 & 0.95 & 1.06 & 1.03 & 0.87 & 0.92 & 0.91 & 1.12 & \\
\hline
\end{tabular}

The maximum values were recorded from plants received $28.6 \mathrm{KgK}_{2} \mathrm{Oha}^{-1}+2 \% \mathrm{~K}_{2} \mathrm{O}$ in $2^{\text {nd }}, 6^{\text {th }}$ and $7^{\text {th }}$ cuts in both years and $5^{\text {th }}$ cut in the second year, while plants received $57.14 \mathrm{KgK}_{2} \mathrm{O} / \mathrm{ha}$ and $28.6 \mathrm{KgK}_{2} \mathrm{O} / \mathrm{ha}+2 \% \mathrm{~K}_{2} \mathrm{O}$ gave the highest protein values in $1^{\text {st }}, 4^{\text {th }}, 8^{\text {th }}$ and $9^{\text {th }}$ cuts in second year and $3^{\text {rd }}$ cut in both years, with insignificant difference. However plants treated by $28.6 \mathrm{KgK}_{2} \mathrm{Oha}^{-1}+2 \% \mathrm{~K}_{2} \mathrm{O}$, $57.14 \mathrm{KgK}_{2} \mathrm{O} /$ ha and $28.6 \mathrm{KgK}_{2} \mathrm{O} /$ ha gave the highest values in $8^{\text {th }}$ and $9^{\text {th }}$ cuts in the first season with insignificant differences. Similar results was recorded by Zizy and Awad (2018) who found that potassium foliar application significantly increased protein content by increasing rate of $\mathrm{K}^{+}$in mono-cut Egyptian clover under saline soil.

\section{Regression Analysis:}

Fig. (1) Showed a linear relationship between potassium fertilizer rates (soil applications, $\mathrm{K}_{2} \& \mathrm{~K}_{3}$ only) and fresh forage weight/ha during the potassium fertilizer rates from 0.00 to $57.14 \mathrm{KgK}_{2} \mathrm{O} / \mathrm{ha}$. The linear regression equation showed that as potassium fertilizer rate increased by one unit/ha, fresh forage weight/ha increased by 1.14 ton/ha within the used potassium fertilizer rates. The highest fresh forage weight/ha (124.210) was produced under the rate of $57.14 \mathrm{KgK}_{2} \mathrm{O} / \mathrm{ha}$. That relationship was described by the following equation: $Y=96.858+1.139 X$ with coefficient of determination $\left(\mathrm{R}^{2}\right)$ equal 1 .

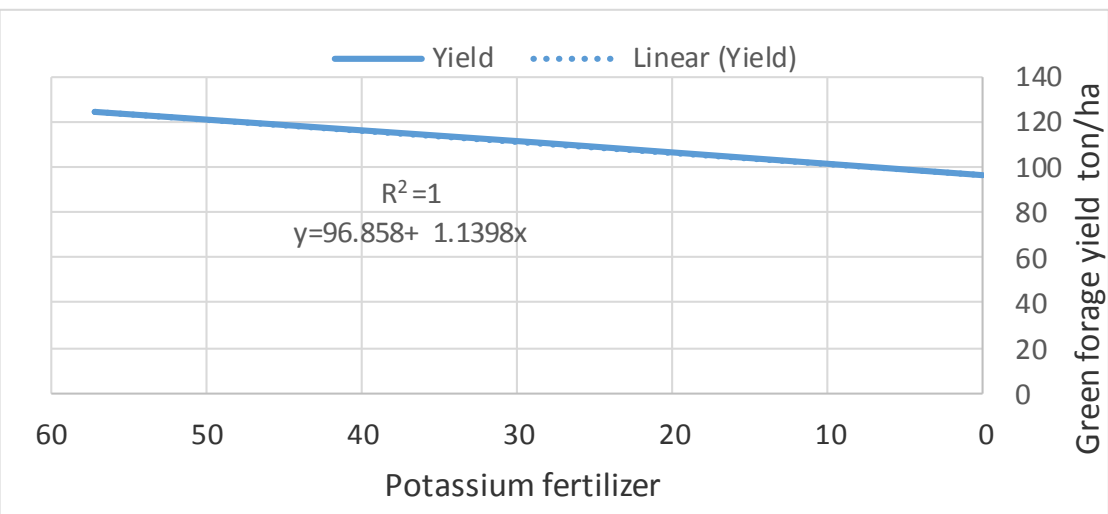

Fig. 1. Regression between potassium rates (soil applications) and fresh forage weight (ton/ha) as average of two years. 
Fig. (2) Showed that relationship between potassium fertilizer rates (foliar applications, $\mathrm{K}_{4}$ \& $\mathrm{K}_{5}$ ) and fresh forage weight/ha was a linear relationship. The relationship was described by the following equation: $Y=96.724+3.112 X$ and the coefficient of determination $\left(\mathrm{R}^{2}\right)$ was 0.99 . The simple linear regression equation showed that as potassium fertilizer rate increased by one unit/ha, fresh forage weight/ha increased by 3.112 ton/ha within the used foliar potassium fertilizer rates. The highest fresh forage weight/ha (103.080) was found at the rate of $2 \% \mathrm{~K}_{2} \mathrm{O} / \mathrm{ha}$.

As for the regression relationship between potassium fertilizer rates $(28.6 \mathrm{~K} 2 \mathrm{O} /$ ha with 1 and 2 foliar applications, $\mathrm{K}_{6} \& \mathrm{~K}_{7}$ )) and fresh forage weight/ha (Fig. 3) the results showed that this relationship was a linear relationship described by this equation $Y=99.018+14.498 X$, with a coefficient of determination (R2) equal 0.937. The highest fresh forage weight /ha (125.850 ton/ha) was produced under treatment of $28.6 \mathrm{~K}_{2} \mathrm{O} / \mathrm{ha}+2 \% \mathrm{~K}_{2} \mathrm{O}$ foliar.

\section{Correlations between the studied traits:}

The simple correlation coefficients were calculated between 10 alfalfa traits and the results are presented in Table (23). The results showed highly positive significant correlation coefficients between all studied traits except the relations between sodium (\%) and the other nine traits which were highly significant negative relationships. Within the positive correlation coefficients, the values of the correlation coefficients ranged from 0.999 for the correlation between fresh forage yield and dry forage yield and 0.850 for the correlation between fresh forage yield and potassium (\%). On the other hand the correlation coefficients between the sodium $(\%)$ and the other traits ranged from 0.992 with leaf/stem ratio and -0.925 with dry forage yield.

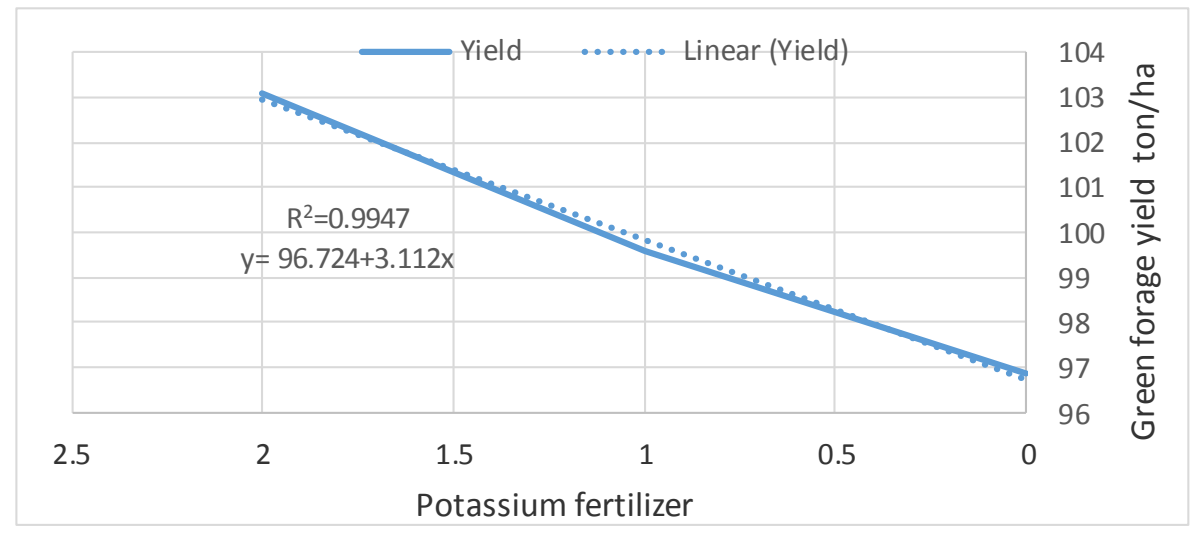

Fig. 2. Regression between foliar potassium fertilizer rates and fresh forage weight/ha (ton) as average of two years.

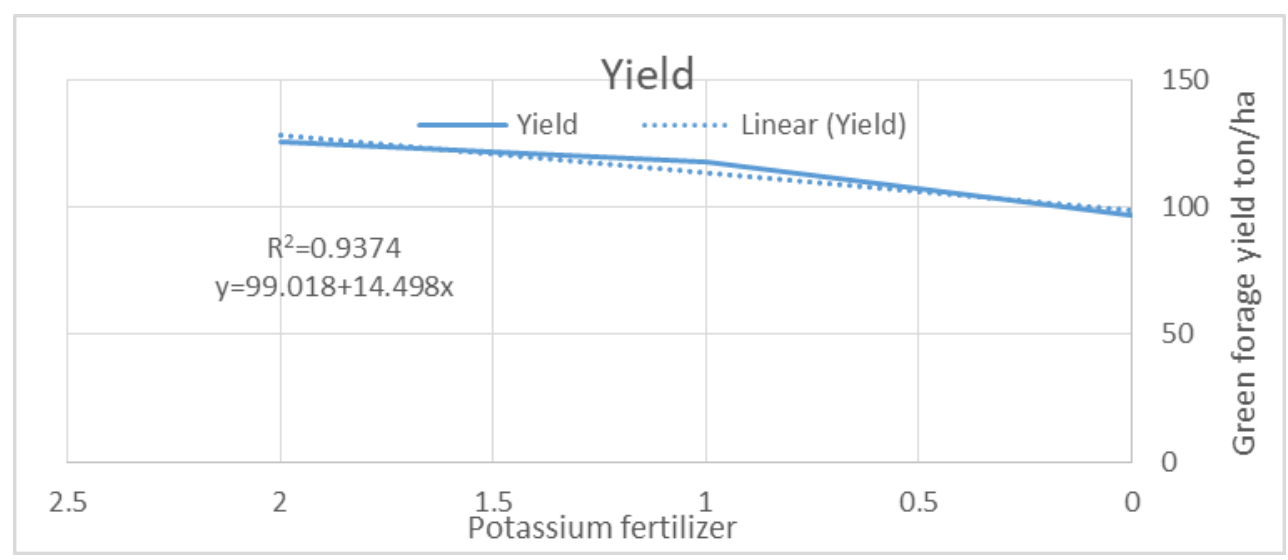

Fig. 3. Regression between potassium levels $\left(\left(28.6 \mathrm{~K}_{2} \mathrm{Oha}^{-1}+\right.\right.$ foliar applications) and fresh forage weight (ton/ha) as average of two years. 
Profitability assessment:

Data illustrated in Table (24) show the total costs of input variables for the experimental treatments (all costs of cultivation process), total input costs and outputs, while net income and the investment ratio for the tested treatments are presented in Tables (25 and 26).

Table 23: Correlation Coefficient estimated between effects of potassium treatments on physiological aspects of alfalfa.

\begin{tabular}{|c|c|c|c|c|c|c|c|c|c|c|}
\hline & $\begin{array}{c}\text { Plant } \\
\text { height }\end{array}$ & $\begin{array}{l}\text { Leaf/ } \\
\text { stem } \\
\text { ratio }\end{array}$ & $\begin{array}{c}\text { Green } \\
\text { forage } \\
\text { yield }\end{array}$ & $\begin{array}{c}\text { Dry } \\
\text { matter } \\
\% \\
\end{array}$ & $\begin{array}{c}\text { Dry } \\
\text { forage } \\
\text { yield }\end{array}$ & RWC & $\mathbf{K} \%$ & $\mathrm{Na} \%$ & $\mathbf{K}^{+} / \mathbf{N a}^{+}$ & $\begin{array}{c}\text { Crude } \\
\text { Protein } \\
\% \\
\end{array}$ \\
\hline plant height & 1.000 & $0.996^{* *}$ & $0.949 * *$ & $0.951 * *$ & $0.955^{* *}$ & $0.973^{* *}$ & $0.967 * *$ & $-0.990 * *$ & $0.992 * *$ & $0.972 * *$ \\
\hline Leaf/stem ratio & & 1.000 & $0.969 * *$ & $0.964 * *$ & $0.975 * *$ & 0.981 ** & $0.944 * *$ & $-0.992 * *$ & $0.982 * *$ & $0.986 * *$ \\
\hline Green forage yield & & & 1.000 & $0.956 * *$ & $0.999 * *$ & 0.970 ** & $0.850 *$ & $-0.959 * *$ & $0.919 * *$ & $0.974 * *$ \\
\hline Dry matter $\%$ & & & & 1.000 & $0.965 * *$ & 0.916 ** & $0.855^{*}$ & $-0.925 * *$ & $0.908 * *$ & $0.982 * *$ \\
\hline Dry forage yield & & & & & 1.000 & $0.972 * *$ & $0.857 *$ & $-0.962 * *$ & $0.924 * *$ & $0.980 * *$ \\
\hline RWC & & & & & & 1.000 & $0.917 * *$ & $-0.989 * *$ & $0.965 * *$ & $0.958 * *$ \\
\hline Potassium\% & & & & & & & 1.000 & $-0.956^{* *}$ & $0.987 * *$ & $0.888^{* * *}$ \\
\hline Sodium\% & & & & & & & & 1.000 & $-0.990 * *$ & $-0.963 * *$ \\
\hline $\mathrm{K}^{+} / \mathrm{Na}^{+}$ & & & & & & & & & 1.000 & $0.943 * *$ \\
\hline Crude Protein \% & & & & & & & & & & 1.000 \\
\hline
\end{tabular}

*: significant at $\mathrm{p} \leq 0.05, * *$ : significant at $\mathrm{p} \leq 0.01$

Table 24: Input production items and output of the experimental work for alfalfa crop in four seasons growing years 2015/2016 and 2016/2017

\begin{tabular}{|c|c|c|c|}
\hline Items & Treatments & Treatments unit & Unit price (L.E) \\
\hline \multicolumn{4}{|l|}{ Inputs } \\
\hline \multicolumn{4}{|l|}{ Mineral fertilizers } \\
\hline $\mathrm{N}$ & 47.62 & Kg N/ha & 13.20 \\
\hline $\mathrm{P}_{2} \mathrm{O}_{5}$ & 147.62 & $\mathrm{Kg} \mathrm{P}_{2} \mathrm{O}_{5} / \mathrm{ha}$ & 6.06 \\
\hline \multicolumn{4}{|l|}{$\mathrm{K}_{2} \mathrm{O}$} \\
\hline K1 & Zero & - & - \\
\hline $\mathrm{K} 2$ & 57.14 & $\mathrm{Kg} \mathrm{K}_{2} \mathrm{O} / \mathrm{ha}$ & 14.58 \\
\hline K3 & 28.57 & $\mathrm{Kg} \mathrm{K}_{2} \mathrm{O} / \mathrm{ha}$ & 14.58 \\
\hline K6 & 28.57 & $\mathrm{Kg} \mathrm{K}_{2} \mathrm{O} / \mathrm{ha}$ & 14.58 \\
\hline K7 & 28.57 & $\mathrm{Kg} \mathrm{K}_{2} \mathrm{O} / \mathrm{ha}$ & 14.58 \\
\hline \multicolumn{4}{|l|}{ Foliar application } \\
\hline K1 & Zero & & \\
\hline K4 & $1 \%(4.75 \mathrm{~L} / \mathrm{ha})$ & Potassin 30\%K & 50 \\
\hline K5 & $2 \%(9.50 \mathrm{~L} / \mathrm{ha})$ & Potassin 30\% K & 50 \\
\hline K6 & $1 \%(4.75 \mathrm{~L} / \mathrm{ha})$ & Potassin $30 \% \mathrm{~K}$ & 50 \\
\hline K7 & $2 \%(9.50 \mathrm{~L} / \mathrm{ha})$ & Potassin 30\% K & 50 \\
\hline Seeds & 47.62 & Kg seeds/ha & 140 \\
\hline Land preparation* & & LE per hectare & 650 \\
\hline Labour** & & & 3880 \\
\hline Other costs*** & & & 2800 \\
\hline Outputs alfalfa yield & & & \\
\hline Winter & & & 120 \\
\hline Spring & & $\mathrm{t} / \mathrm{ha}$ & 180 \\
\hline Summer & & & 250 \\
\hline Autumn & & & 200 \\
\hline
\end{tabular}

* Rent of agricultural machines ** Cultivation, irrigation, fertilization, Thinning, etc.

$* * *$ Land rent, transportation of seeds, fertilizers, etc. Irrigation and drainage systems conservation,. etc. 
Table 25: Experimental total outputs (LE/ha) for four seasons during the two years 2015/16 and 2016/17.

\begin{tabular}{|c|c|c|c|c|c|c|c|c|c|c|c|}
\hline \multirow[b]{2}{*}{ Treatments } & \multicolumn{5}{|c|}{ Winter } & \multicolumn{5}{|c|}{ Spring } & \\
\hline & 2016 & 2017 & Mean & Unit & $\begin{array}{l}\text { Output } \\
\text { LE/ha }\end{array}$ & 2016 & 2017 & Mean & Unit & $\begin{array}{r}\text { Output } \\
\text { LE/ha }\end{array}$ & \\
\hline $\mathrm{K} 1$ & 13.78 & 20.18 & 16.98 & 120 & 2037.6 & 22.81 & 31.48 & 27.15 & 180 & 4887.0 & \\
\hline $\mathrm{K} 2$ & 15.98 & 23.41 & 19.70 & 120 & 2364.0 & 29.47 & 36.62 & 33.05 & 180 & 5949.0 & \\
\hline K3 & 14.58 & 22.44 & 18.51 & 120 & 2221.2 & 24.88 & 32.06 & 28.47 & 180 & 5124.6 & \\
\hline K4 & 14.21 & 21.33 & 17.77 & 120 & 2132.4 & 23.28 & 29.03 & 26.16 & 180 & 4708.8 & \\
\hline K5 & 14.63 & 21.86 & 18.25 & 120 & 2190.0 & 23.43 & 30.12 & 26.78 & 180 & 4820.4 & \\
\hline K6 & 14.85 & 22.79 & 18.82 & 120 & 2258.4 & 26.45 & 34.17 & 30.36 & 180 & 5464.8 & \\
\hline $\mathrm{K} 7$ & 15.69 & 24.12 & 19.91 & 120 & 2389.2 & 29.47 & 37.06 & 33.27 & 180 & 5988.6 & \\
\hline \multirow[t]{2}{*}{ Treatments } & \multicolumn{6}{|c|}{ Summer } & \multicolumn{4}{|c|}{ Autumn } & Total \\
\hline & 2016 & 2017 & Mean & Unit & $\begin{array}{r}\text { Output } \\
\text { LE/ha }\end{array}$ & 2016 & 2017 & Mean & Unit & $\begin{array}{r}\text { Output } \\
\text { LE/ha }\end{array}$ & $\begin{array}{l}\text { output } \\
\text { LE/ha }\end{array}$ \\
\hline K1 & 28.76 & 39.73 & 34.25 & 250 & 8562.5 & 16.23 & 23.33 & 19.78 & 200 & 3956.0 & 19443.1 \\
\hline K2 & 38.48 & 50.99 & 44.74 & 250 & 11185.0 & 23.22 & 30.37 & 26.80 & 200 & 5360.0 & 24858.0 \\
\hline K3 & 33.42 & 46.63 & 40.03 & 250 & 10007.5 & 19.88 & 27.15 & 23.52 & 200 & 4704.0 & 22057.3 \\
\hline K4 & 29.40 & 41.00 & 35.20 & 250 & 8800.0 & 17.13 & 24.01 & 20.57 & 200 & 4114.0 & 19755.2 \\
\hline K5 & 30.71 & 43.05 & 36.88 & 250 & 9220.0 & 17.93 & 24.44 & 21.19 & 200 & 4238.0 & 20468.4 \\
\hline K6 & 36.60 & 49.29 & 42.95 & 250 & 10737.5 & 21.58 & 29.97 & 25.78 & 200 & 5156.0 & 23616.7 \\
\hline K7 & 38.67 & 51.64 & 45.16 & 250 & 11290.0 & 23.08 & 31.27 & 27.18 & 200 & 5436.0 & 25103.8 \\
\hline
\end{tabular}

The results indicated that the highest total output values $(25103.8$ and $24858.0 \mathrm{LE} / \mathrm{ha})$ were obtained from the treatment $\mathrm{K} 7$ (28. $6 \mathrm{KgK}_{2} \mathrm{O} / \mathrm{ha}+2 \%$ potassium foliar application) followed by $\mathrm{K} 2$ (57.14 $\left.\mathrm{KgK}_{2} \mathrm{O} / \mathrm{ha}\right)$. The results, also, revealed that the highest outputs (11290.0 and $11185.0 \mathrm{LE} / \mathrm{ha})$ were obtained under the same above treatments in the summer season for the two experimental years, while the lowest values of total outputs were always obtained from control treatment of K1 (19443.1 LE/ha) and K4 (19755.2 EL/ha) and in the winter season for the same treatments K1 (2037.6) and K4 (2132.4 LE/ha)

On the other hand total net income and investment ratio values were incorporated with the highest output values in the descending order K7> $\mathrm{K} 2>\mathrm{K} 6>\mathrm{K} 3>\mathrm{K} 5>\mathrm{K} 4 \geq \mathrm{K} 1$. The data revealed that highest values of net income and investment ratio were 8691.29 LE/ha and 1.53 for K7 and 8504.94 LE/ha and 1.52 for K2 respectively
Finally from the obtained data it could be concluded that under saline calcareous soil conditions the addition of $28.6 \mathrm{KgK}_{2} \mathrm{O} / \mathrm{ha}$ with fertilizer application of $2 \% \mathrm{~K}_{2} \mathrm{O}$ as potassin $30 \% \mathrm{~K}^{+}$ followed by addition of $57.14 \mathrm{KgK} 2 \mathrm{O} / \mathrm{ha}$ significantly obtained the highest values of alfalfa yield with insignificant difference. These results were accompanied with the highest values of output, net income and the investment ratio.

\section{CONCLUSION}

From the obtained results in this study, it could be concluded that, alfalfa production can be maximized by adding $28.6 \mathrm{KgK}_{2} \mathrm{O} / \mathrm{ha}$ as soil dressing in combination with $2 \% \mathrm{~K}_{2} \mathrm{O}$ (potassin $30 \% \quad \mathrm{~K}_{2} \mathrm{O}$ ) as foliar application under saline calcareous soil conditions at Nubaria region. In addition these results were incorporated with the highest values of output, net income and the investment ratio. 


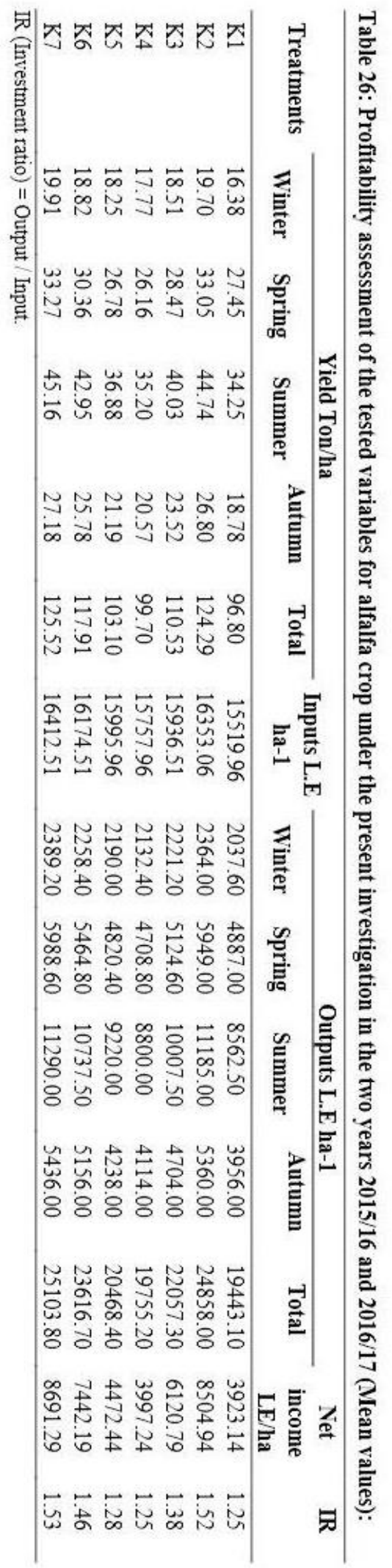




\section{REFERENCES}

Abdo Fatma, A. and N.A Anton, 2009. Physiological response of sesame to soil moisture stress and potassium fertilization in sandy soil. Fayoum J. Agric. Res \& De. 23(1): 88-111.

Almeida, D.M.; Oliveira, M.M.; Saibo, N.J.M. 2017, Regulation of $\mathrm{Na}^{+}$and $\mathrm{K}^{+}$homeostasis in plants: Towards improved salt stress tolerance in crop plants. Genet. Mol. Biol., 40: $326-345$.

Annunziata, M.G.; Ciarmiello, L.F.; Woodrow, P.; Maximova, E.; Fuggi, A.; Carillo, P. (2017) Durum wheat roots adapt to salinity remodeling the cellular content of nitrogen metabolites and sucrose. Front. Plant Sci., 7: 2035.

Ashraf, M.; Harris, P.J.C. (2013) Photosynthesis under stressful environment: An overview. Photosynthetic, 51: 163-190.

Ashraf, M. and J.C. Harris (2004). Potential biochemical indicators of salinity tolerance in plants. Plant sci., 166: 3-166.

A.O.A.C. (1990) Official Methods of Analysis, 15th ed., Association of Official Analytical Chemists, Inc., Virginia, USA, 770-771.

Bertrand, A., Dhont, C., Bipfubusa, M., Chalifour, F.P., Drouin, P. and Beauchamp, C.J. (2015) Improving Salt Stress Responses of the Symbiosis in Alfalfa Using Salt-Tolerant Cultivar and Rhizobial Strain. Applied Soil Ecology: 87, 108-117.

Bhattarai,S.,D. Biswas, Y. BiFu and B. Bill (2020). Morphological, Physiological, and Genayic Responses to Salt Stress in Alfalfa.Agronomy, $1-15$.

Black, C.A. (Ed.) (1965). Methods of Soil Analysis, Parts and 2.Amer. soc. Agron No. 9. Madioon. Wioconoin, USA.

Castroluna, A. (2009). Fisiología de la germinación y crecimiento de tres variedades de Medicago sativa bajo condiciones de estrés abiótico. Grade Thesis, National University of San Luís, San Luís, Argentina, 98.

Carillo, P.; Cirillo ,C.; De Micco, V.; Arena, C.; De Pascale,S.;Rouphaelb, Y. (2019). MorphoAnatomical, physiological and biochemical adaptive responses to saline water of Bougainvillea spectabilis Willd. Trained to different canopy shapes. Agric. Water Manag., 212: 12-22.

Chapman, H.D. and P.F. Pratt (1978). Methods of analysis for soil, plant and waters. Univ. of Californiae. Div. Agri. Sci., Priced Publication4043.
Chawla S., S. Jain and V. Jain (2013). Salinity induced Oxidative stress and antioxidant system in salt-tolerant and salt-sensitive cultivars of rice (Oryza sativa L.). J. Plant Biochem. Biotechnol., 22: 27-34.

El-Sharkawy,M. S., T. R. El-Beshsbeshy, E. K. Mahmoud, N. I. Abdelkader, R. M. AL-Shal and A. M. Missaoui (2017) Response of Alfalfa under Salt Stress to the Application of Potassium Sulfate Nanoparticles. American Journal of Plant Sciences, 8: 1751-1773.

Eman, Y., Bijanzadeh, E., Naderi, R. and Edalat, M. (2009) Effect of Salt Stress on Vegetative Growth and Ion Accumulation of Two Alfalfa (L.) Cultivars. Desert, 14: 163-169.

Flowers, T. J., P. F. Troke and A. R. Yeo (1977). The mechanism of tolerance in halophytes. Annu. Rev. Plant Physiol., 28: 89-121.

Gill, S. S. and N. Tuteja (2010). Reactive oxygen species and antioxidant machinery in abiotic stress tolerance in crop plants. Plant Physiol. Biochem., 48: 909-930.

Goplen, B.P.; Baenziger, H.; Bailey, L.D.; Gross, A.T.H.; Hanna, M.R.; Michaud, R.; Richards, K.W.; Waddington, J. (1982) Agriculture Canada: Growing and Managing Alfalfa in Canada; Publication 1705/E; Agriculture Canada: Ottawa, ON, Canada,.

Greenway H. and R, Munns (1980). Mechanisms of salt tolerance in no halophytes. Annu. Rev. Plant Physiol., 31: 149-190.

Hasanuzzaman, M.; M. H. M. Borhannelddin, K. Kamrun, Md. H. Shahadat 'A. Juhayer, Md. Shahadat, Abdul Awal M. Chowdhury, Moumita and Masayuki Fujita. (2018). A vital Regulator of plant Responses and Tolerance to Abiotic stresses. Agronomy Review 8, 31-29.

Howkesford, M.; Horst, W.; Kichey, T.; Lambers, H.; Schjoerring, J.; SkrumsagerMoller, I.; White, P. 2012 Function of macronutrients. In Marschner's Mineral Nutrition of Higher Plants; Marschner, P., Ed.; Academic Press: London, UK,; pp. 135-189.

Heba. G. Ali and Mary E. Nashed (2017). Evaluation of seven barely genotypes to Potassium Fertilization in Saline soil. Proceeding of the $15^{\text {th }}$ International Symposium on Biocontrol and Biotechnology, Hurgada, Egypt. 82-94.

Khorshidi, M. B., Yarnia, M. \& Hassanpanah, D. (2009). Salinity effect on nutrient accumulation in alfalfa shoots in hydroponic condition. J. Food Agric. Environ. 7: 787-790.

Lazcano-Ferrat, I. and C. J. Lovatt, 1999. Relationship between relative water content, nitrogen pools and growth of phaseolus vulgaris, L. and P. acutifolius during water deficit. Crop Sci., 39(2):467-457. 
Montgomery, D. C. and E. A. Peck (1982). Introduction to Linear Regression Analysis. John Wiley, NY, USA.

Mousa, M. E., I, A. Hanna and Z. M. Marei,(1996). Evaluation of some Alfafla (Medicago sativa L.) cultivars for growth and yield in sandy soils at north east of Egypt. Zagazig J. Agric. Res. 23 (1): $29-49$.

Mueller S.C. and L.R. Teuber (2007) Alfalfa Growth and Development. Univ.of California, Division of Agriculture and Natural Resources, Chapter 3: 1-9.

Munns, R. (2002). Comparative physiology of salt and water stress. Plant Cell Environ., 25: 239-250.

Munns, R.; James, R.A.; A. Lauchli, (2006) Approaches to increasing the salt tolerance of wheat and other cereals. J. Exp. Bot., 57, 1025-1043.

Munns, R.; M. Tester, (2008) Mechanisms of salinity tolerance. Annu. Rev. PlantBiol. 59: $651-681$.

SAS, .2014. SAS: Business Analytics and Business Intelligence Software. SAS Inst. http://www.sas.com/en_us/home.html.

Schachtman, D. and W.H. Liu, (1999) Molecular pieces to the puzzle of the interaction between potassium and sodium uptake in plants. Trends Plant Sci., 4, 282-287.

Shabala S. and T.A. Cun, (2008). Potassium transport and plant salt tolerance. Plant.133: 651-669.

Snedecor,G. W. and. W. C. Cochran (1980) Statistical Methods, Oxford and IBH publishing 7th ed. Iowa State univ. Press, Iowa USA.
Steel, R. F. and J. H. Torrie (1980). Principles and Procedures of Statistical $2^{\text {nd }}$ ed. McGrawHill Book Co. Inc. NEW York.

Su, L.; Dai, Z.; Li, S.; Xin, H. (2015 a) A novel system for evaluating drought-cold tolerance of grapevines using chlorophyll fluorescence. BMC Plant Biol., 15: 82-85.

Su, Y.; Luo, W.; Lin, W.; Ma, L.; Kabir, M.H. (2015 b) Model of cation transportation mediated by high-affinity potassium transporters (HKTs) in higher plants. Biol. Proced. Online, 17, 1-8.

Tanji, K.K. (1990) Nature and Extent of Agricultural Salinity. In Agricultural Salinity Assessment and Management; Tanji, K.K., Ed.; American Society Civil Engineers: New York, NY, USA,; pp. 1-13.

Tuteja, N. (2007) Mechanisms of high salinity tolerance in plants. Methods Enzymol, 428: 419-438.

Undersander D.,H.H.Marvin, P.Vassalotti and D. Cosgrove (2011). Alfalfa Germination and Growth, National Alfalfa and Forage Alliance (NAFA), WW W.alfalfa.org.

Wang, W. B; Vinocur and A. Altman (2003). Plant responses to drought, salinity and extreme temperatures towards genetic engineering for stress tolerance, Planta, 21: 1-14.

Zizy M. Abbas and A. M. Awad (2018). Effect of Potassium Foliar Application on Productivity and Quality of Mono-Cut Egyptian Clover under Saline Soil. Egypt. J. Agron., 40: 155163.

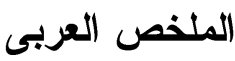

\section{الاستجابة الفسيولوجية وانتاجية البرسيم الحجازى للاضافة الارضية ورش البوتاسيوم تحت ظروف الاراضى الجيرية الملحية

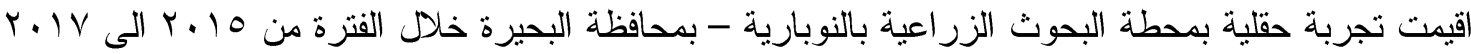

لدر اسة الاستجابة الفسيولوجية و انتاجية البرسيم الحجازى (صنف نوبارية () لاضافة معدلات التسميد الارض صفر،

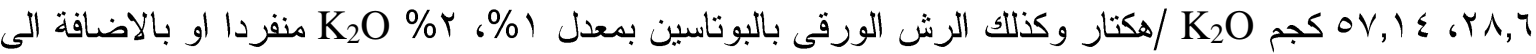
Y, T كجم/هكتار تحت ظروف الارض الجيرية الملحية وثم اخذ 1 1 حشة (وحشات/سنة) لتقدير صفات النمو و انتاجية البرسيم الحجازى وكذللك بعض الصفات الفسيولوجية. ويمكن تلخيص النتائج لكلا السنتين كالأتى: 
- كان لمعاملات التسميد البوتاسى تأثير ا معنويا لجميع الصفات فى جميع الحشات ماعدا الحشة الاولى و التاسعة فى

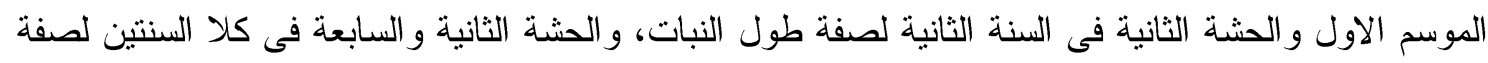

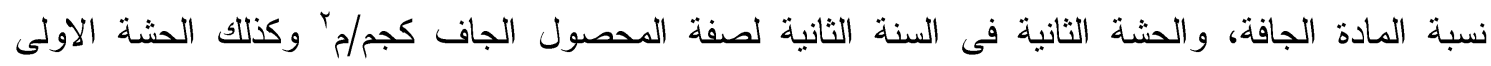

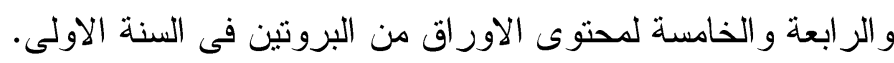

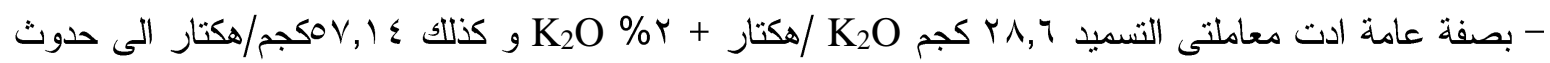
زيادة معنوية لكل من طول النبات، نسبة الاوراق/ساق، محصول العلف الاخضر و الجاف كجم/م و المحصول

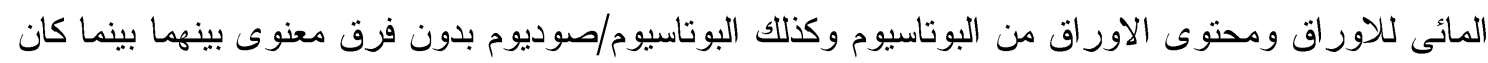

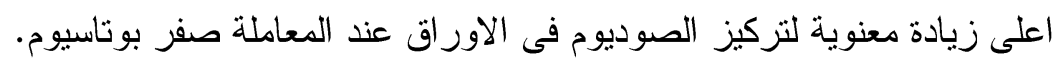

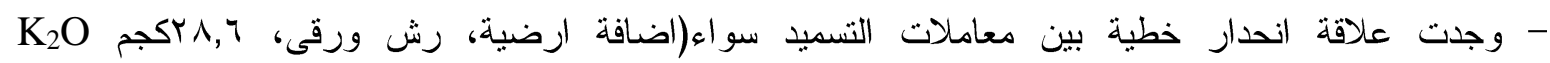

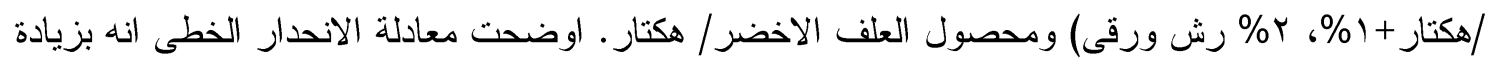

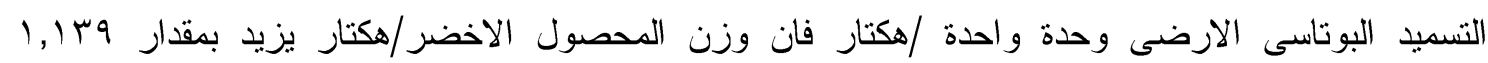

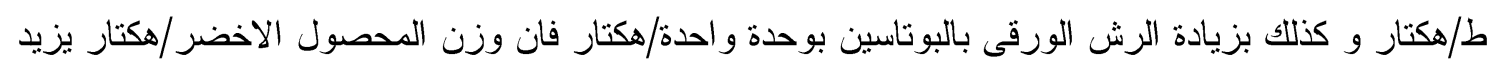

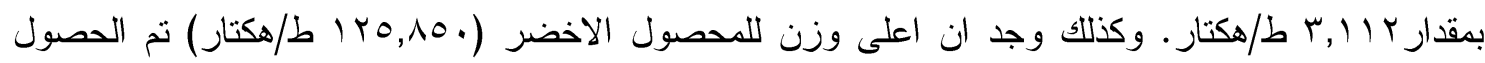
عليه عند المعاملة r ا كجم - هناك علاقة ارتباط موجبة ومعنوية بين جميع الصفات تحت الدراسة ماعدا العلاقة بين محتوى الصوديوم مع باقى الصفات حيث انها علاقة ارتباط سالبة ومعنوية.

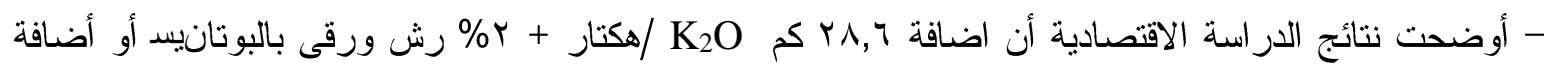

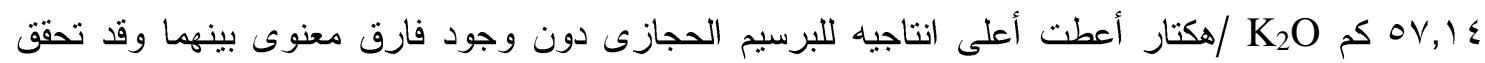
أعلى دخل مزرعى وصافى الدخل المزرعى وأعلى عائد استثمارى تحت نفس المعامله التى أعطت أعلى ألى انتاجيه محصوليه للبرسيم الحجازى.

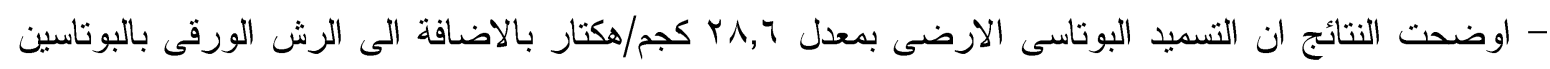

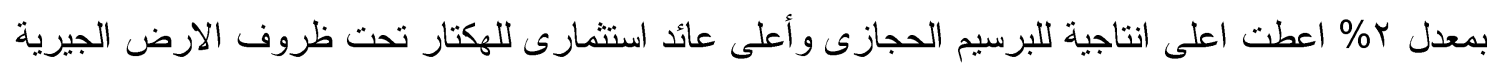

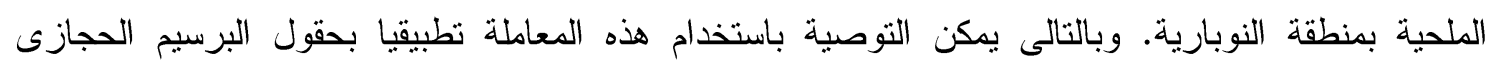
المزروع فى الار اضى المماثلة لتلك التى فى النوبارية لتحسين الانتاجية. 